\title{
Emerging Experimental Strategies for Studies on Inhomogeneous Nanomaterials. Exploring Electronics, Atomic Structure and Properties by Synchrotron X-Ray-Based Techniques
}

\author{
Félix G. Requejo \\ Instituto de Investigaciones Fisicoquímicas Teóricas y Aplicadas (INIFTA), CONICET y FCE, UNLP, La Plata, Argentina. \\ E-mail: requejo@inifta.unlp.edu.ar
}

\begin{abstract}
Through representative examples, we illustrate the strategic value of synchrotron X-ray-based techniques for nanomaterial characterization, especially for inhomogeneous nanomaterials. These examples are used to describe opportunities, motivations for new questions and possible reexamination of older topics, not only due to the proven success of the scientific instrumentation, but also because the extraordinary specific advantages offered by the new X-ray micro and nanoprobes techniques.
\end{abstract}

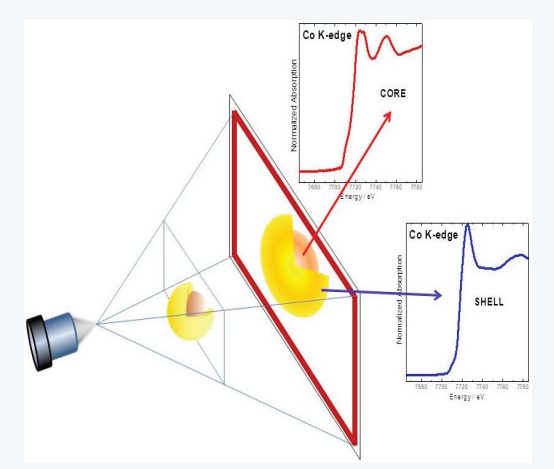

Keywords:

synchrotron radiation, microprobes and nanoprobes, nanomaterials, nanoparticles

\section{Focus of the review}

This present review is aimed at describing recent improvements for nanomaterial characterizations strategies using a meaningful set of scientific cases related to the demanding understanding of inhomogeneous nanomaterials structure and electronics. We present a set of emerging techniques based on X-ray interaction with matter, paying special attention to $\mathrm{X}$-ray micro and nanoprobes tools, discussing their impact in the nanoscience research field.

\section{Introduction}

\section{Nanomaterial characterizations and synchrotron radiation-based studies}

The demand for advanced characterization in nanomaterials is a neck of the bottle for the increasing challenge in the rational design of new nanomaterials and nanocomposites. Typical limitations are, in general, associated with chemical speciation at different regions of nanomaterials (distinguishing surface from volume contributions), in situ determinations controlling the environment at very low time-scales, and/or sensitivity for the detection of small concentrations of the species (atomic clusters or individual atoms). Undoubtedly, no single experimental approach can be enough to elucidate any relevant question about atomic and electronic structure, responsible for chemical, mechanical, magnetic or any other physical properties. Different "cocktails" of experimental strategies became mandatory for that purpose, where new and fascinating instrumental developments are being successfully applied. At this point, the synchrotron-based techniques emerge as one of the more relevant solutions to attend the growing demand for advanced nanomaterial characterizations. 
In particular, the progress of synchrotron sources for X-rays not only evolves in higher brilliance, but also in emittance and coherence, in addition to drastic improvements for insertion devices and scientific instrumentation at end-stations (like detectors or electronics and new architecture for data acquisition). From the beginning, synchrotron facilities for scientific research constitute an attractive opportunity to look for new solutions, and to turn on the imagination for new experimental challenges, pushing beyond the limit of the scientifically known.

Many aspects in nanomaterials can be analyzed taking into account different physical process associated with the interaction of X-rays with matter. X-ray scattering is useful for studying the shape and size of nanomaterials; X-ray absorption is used to determine the electronic structure of empty levels and the local atomic environment of the absorbing atoms; the emission of the excited atoms is associated with the density of occupied electronic levels; selective absorption - considering the spin state - is employed for magnetism studies; and electronic photoemission is very sensible for surface and interphases probing. All these physical processes are being taken into account for the increasing development of advanced instrumentation and techniques (Table 1). An outstanding example for recent advances in instrumentation is the in situ or operando detection modes, in particular to bridge the pressure gap in the XPS technique, represented by the already well-established NAP-XPS technique (Salmeron et al., 2018). Additional developments are being achieved, more recently, devoted to improve time and spatial resolution, which are necessarily associated with $4^{\text {th }}$ generation synchrotron machines (Kwiatek et al., 2019). This review deals precisely with the most recent and emerging improvements, associated with micro and nano-focus progresses.

Table 1. Different X-ray-based synchrotron techniques, their associated physical processes, and their applications for material characterizations.

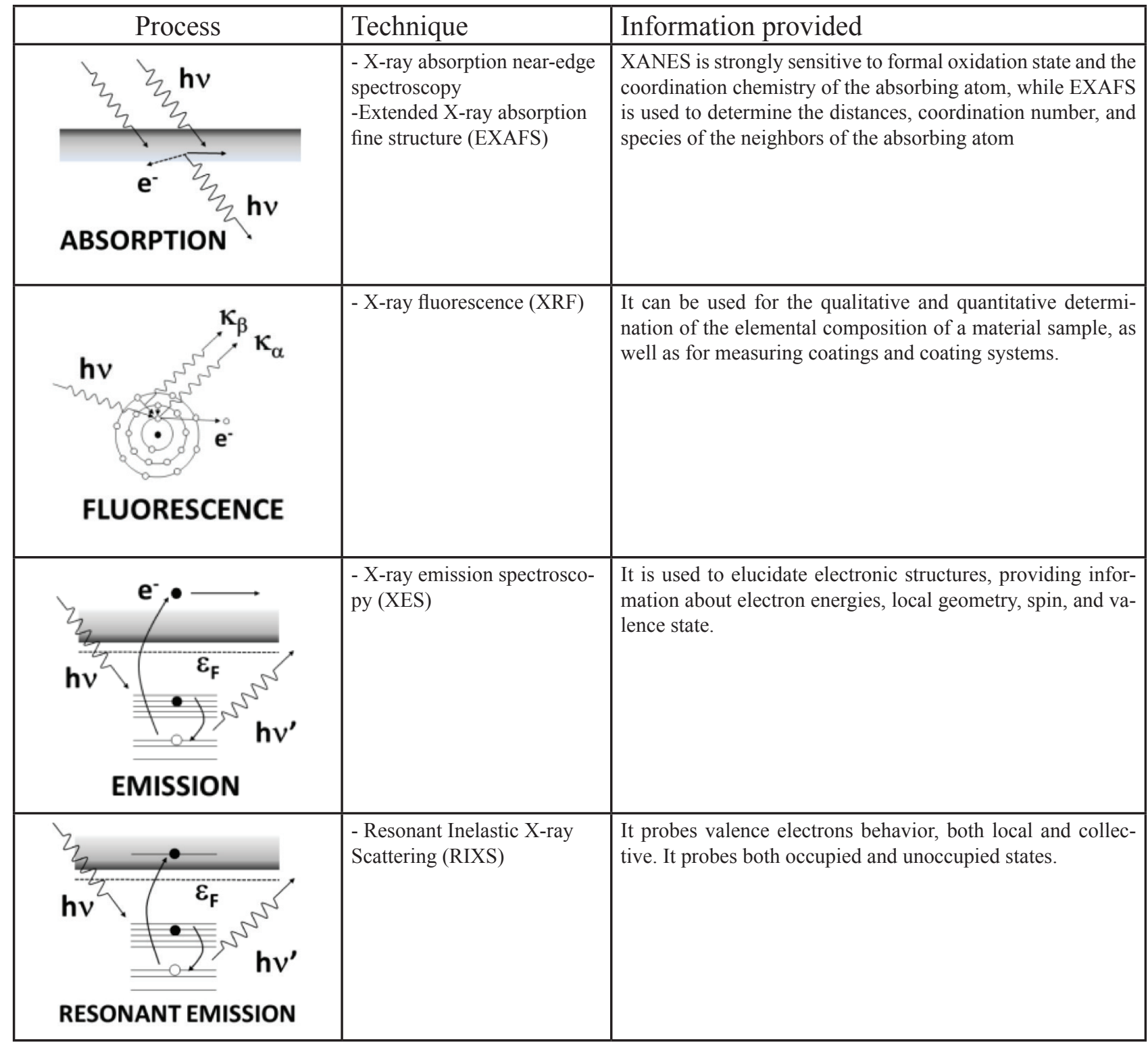




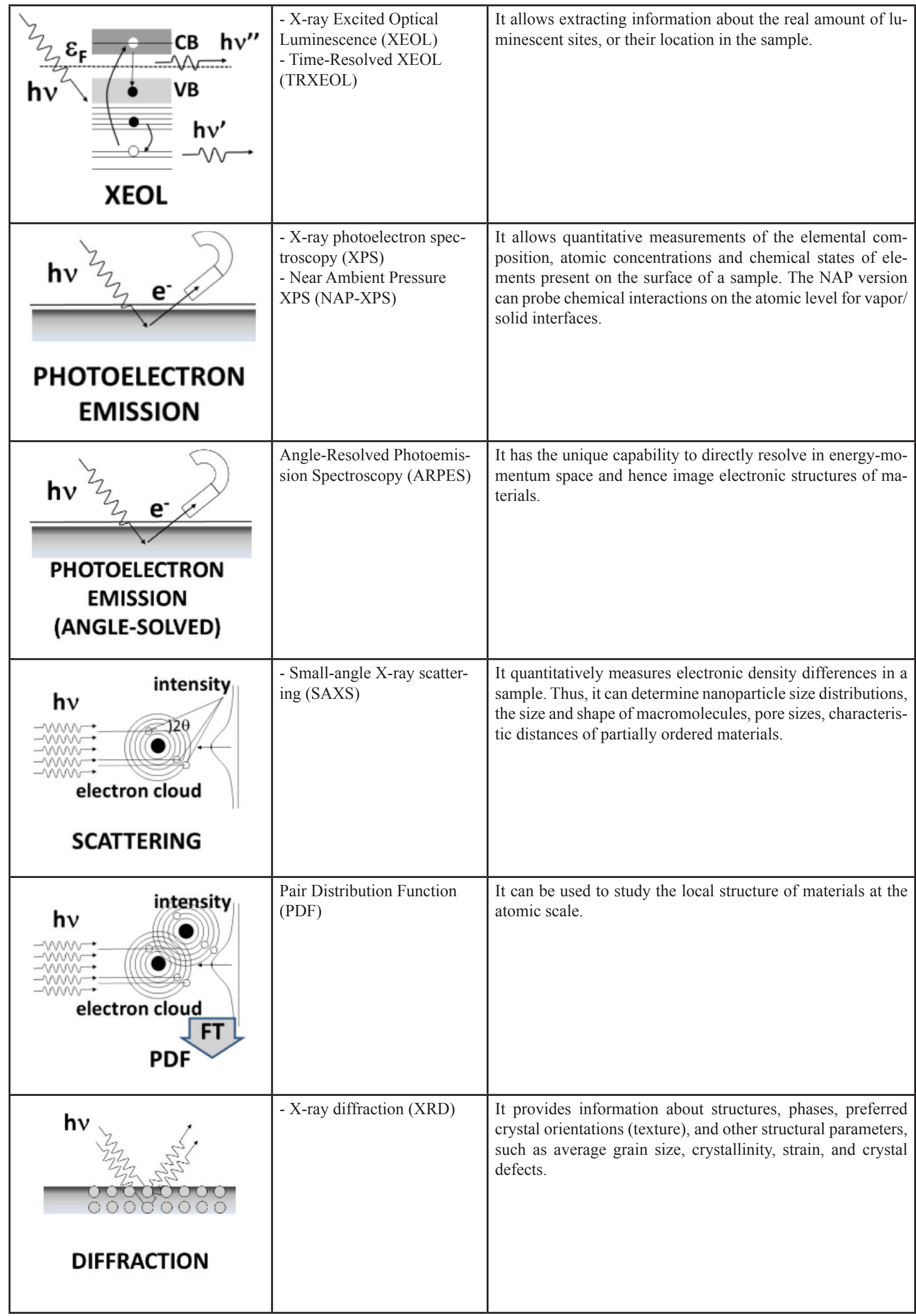




\section{Inhomogeneous nanostructures: interest and characterization}

Among the numerous types of inhomogeneous nanomaterials, core@shell-type (CS) nanostructures are worthy of attention. Those sort of nanoparticles (NPs) present challenges not only because of their synthesis complexity, but also because of their novelty for properties associated with their heterogeneity in composition and/or structure. SC structures can be considered as the simplest possible model to describe inhomogeneity at the nanoscale. Thus, all the efforts in CS characterization represent a progress for understanding more complex structures.

CS NPs are a class of preferred inhomogeneous nanomaterials due to their interesting properties and broad range of applications in catalysis (Guo et al., 2013), biology (Hoskins et al., 2012), materials chemistry (Acebrón et al., 2017), magnetism (Wu L. et al., 2016), energy (Cui et al., 2013) and sensors (Vera et al., 2017). By rationally tuning the cores, as well as the shells, a range of CS NPs can be produced with tailorable properties to play important roles in different physicochemical processes, offering solutions for present technological demands. In general, the definition of the CS NPs can be extended to nanomaterials having different boundary covering (either fully or partially) the inner component, as long as they can be separately identified. At times, they cannot be simply described as a single material surrounded by a homogeneous, different one. In reality, it is possible to find, through few nanometers, a dégradé of compositions and mixtures of chemical elements, structures and species, not easily ascribed to bulk version because of the short periodicity of the atomic arrangements. That peculiar atomic arrangement, finally defines the desired properties for the CS NPs. Thus, the detailed characterization becomes crucial to understand the origin of their properties.

An overview of the available techniques for characterizing CS nanostructures typically includes X-ray diffraction (XRD), scanning electron microscopy (SEM) and transmission electron microscopy (TEM) (Manoj et al., 2015). Even though all these techniques are usually used to determine the size, morphology and homogeneity of the NPs, the first one requires the crystallinity of the phases, while the last two can detect both phases simultaneously. Additionally, SEM usually involves collecting secondary electrons and therefore generates only a surface image, and it is difficult to distinguish the core from the shell. High resolution TEM (HRTEM) enables the simultaneous observation, but not trivial, of lattice borders with different spacing in the core and shell from a single particle. The energy dispersive spectrometry (EDS), as a TEM accessory, provides one of the most powerful methods for determining the chemical distribution of elements with high spatial resolution, including the limitation of the adequate environment for electron detection. Complementary techniques, with high spatial resolution, can be found in electron energy loss spectroscopy (EELS). A variation of those methodologies is represented by high angle annular dark-field STEM (HAADF-STEM), where elements with important contrast in atomic number are required for successful determinations. More recently, images with atomic-resolution can be obtained by aberration-corrected STEM. In another set of more common techniques, we can find different spectroscopies such as UV-Vis that can be employed in specific cases for indirect determinations, which are the most commonly used type of spectroscopy, especially capable of absorbing in the UV-Vis region of the electromagnetic radiation. Infrared spectroscopy is particularly useful for the identification of organic molecules (for organic types of CS nanostructures). Additionally, Raman spectroscopy is widely used for surface characterization, and surface enhanced Raman spectroscopy (SERS) for studying CS NPs containing SERS active metals ( $\mathrm{Au}, \mathrm{Ag}$, and $\mathrm{Cu}$ ).

Synchrotron-based techniques applied to CS NP characterization have been successfully used in recent years. The competence of X-rays to analyze the structure and dynamics of almost all forms of matter has been largely demonstrated. $\mathrm{X}$-rays analysis covers range of lengths that is of interest for nanomaterial characterizations between $10^{-3}$ to about 100 $\mathrm{nm}$, and the time resolution to explore dynamic process from about $10^{-16}$ to $10^{3} \mathrm{~s}$. Several techniques based on scattering, diffraction, spectroscopy and imaging (Table 1) are available nowadays at end-stations in different synchrotrons around the world. The improvements for the different techniques are associated with the increase in intensity, collimation and focusing of the beam, as well as in wavelength tuning. In a synchrotron, charged particles (electrons or positrons) are accelerated and injected into a storage ring, where they get energies from $500 \mathrm{MeV}$ to $8 \mathrm{GeV}$ depending on the size of the ring. As the charged particles are bent around the ring (by magnetic devices), energy is dissipated by electromagnetic radiation, emitted from infrared to X-rays. Essentially, X-ray interacts with most materials, and if the experiment is conducted in a nondestructive way, they can interact selectively with surfaces or penetrate deeply into samples. In addition to this selective region analysis or sensitivity, chemical selectivity is a salient characteristic, which really makes a big difference among the different experimental methodologies, especially in the field of nanoscience. Techniques based on X-ray absorption or emission processes can selectively interact with specific chemical elements just by choosing the correct incident photon energy and/or the regions in which to detect the emitted fluorescence photons. For higher photon energies than the corresponding to the UV region, the radiation produced allows studies under real or extreme conditions of temperature, pressure, and magnetic or electric fields. This is a characteristic that has been already exploited in the last decade for in situ or operando studies (Dong et al., 2018). At the synchrotron sources, radiation is highly intense, highly focused, and strongly polarized. The radiation that can be obtained includes very low energies, even lower than $0.1 \mathrm{keV}$ (considered "soft" X-rays up to about $2 \mathrm{keV}$ ), up to $100 \mathrm{keV}$ (considered as "hard" X-rays from few keV). The intermediate region between 2 and $5 \mathrm{keV}$ is known as the "tender" $\mathrm{X}$-ray window. 
X-ray Absorption spectroscopies (XAS) are a particularly privileged set of techniques, since they allow exploring both electronic and atomic structure in very atomically confined (local) regions. Based on the local scattering of the free electrons produced after the photoelectric effect, the atomic structural aspects around the absorbing atom can be explored by extended X-ray absorption fine structure technique (EXAFS). On the other hand, the electronic structure of the same absorbing element, in particular the density of unoccupied states, is greatly probed in the same process, in the closest energy region to the absorption edge, by X-ray absorption near edge structure (XANES) spectroscopy. Because of the lower energy of the photoelectrons produced in this absorption region, the final estate of the corresponding electronic transition is given by allowed unoccupied electronic states of the same atom or molecule. In contrast to XRD, XAS can also be used for liquid samples or even for gaseous phase characterization, since it does not rely on any long-range order. Thus, XAS provides a very robust methodology for the screening of the crystallization mechanism of amorphous NPs. Phase transformation can be also monitored by XAS experiments from both ex situ and in situ configurations. Additionally, being only sensitive to the local geometrical arrangement of neighboring atoms that surround the absorbing atom, it is possible to study the interaction of the capping agent with the surface of the NP. In particular, EXAFS is appropriated for the screening of the initial crystallization behavior of amorphous NPs by probing structural changes at the atomic-level. EXAFS allowed the precise demonstration of the changes of bond lengths and the stress state of their materials on the basis of a thermal expansion mismatch. The evaluation of the EXAFS data of small NPs provides a decreased coordination number, which involves more sensitivity for parameter analysis for the CS-NP. Thus, the interface effects between core and shell regions, dilatation or contraction of the lattice, Debye-Waller factor, together with an increased static and/ or thermal disorder can be examined. Importantly, in contrast to other experimental techniques based on electronic detection (like conventional XPS or TEM), XAS experiments do not necessarily require operation under ultra-high vacuum. Structural information can be obtained from EXAFS measurements, even though sometimes with lower precision and difficulty in comparison with XRD, it can also be used to distinguish two phases even having similar structure and lattice constants, like in perfectly miscible metals where both phases possess an fcc structure. Considering the symmetry of the different atomic environments, XANES is a technique more sensitive to coordination and bonding environment than, for instance, XPS, since it probes the unoccupied electronic states of atoms and therefore can provide information about the crystal field (octahedral, square pyramidal or tetrahedral) occupied by cations. The complementary use of both XPS and XANES is handy for nanostructures with complex compositions and various possible valence states.

\section{Inhomogeneous nanomaterials characterization using synchrotron X-ray-based techniques}

There is a variety of literature with studies of nanomaterials properties and performance. In particular for NP, because their properties rely heavily on their size, morphology, structure and composition, all those aspects are in general analyzed in depth following a multi-technique approach (Mourdikoudis et al., 2018). The literature specifically devoted to CS-NP seems to be less abundant, where the combination of the inner core and outer shell, dispersion of species and structures on the surface of the NP and interaction at the interface have crucial effects on their properties as well as optical and catalytic activity mainly.

Many advantages and characteristics of X-ray absorption techniques for CS-NP studies were previously described for already installed facilities at synchrotron laboratories (National Nanotechnology Initiative, 2005). More specifically, we will refer now to specific aspects for the direct and indirect determination of relevant parameters, which characterize the fundamental structural and electronic aspects of such nanomaterials, responsible for their physical and chemical properties. Finally, we will refer to general concepts exemplified by a selection of significant applications of x-ray microbeams and nanobeams to materials science research, in particular for emerging challenges in the nanoscience field.

The XANES and EXAFS analyses of the atomic structure and electronic properties of NP sometimes allow discovering spontaneous or unexpected CS-NPs. This is the case, for instance, when the sulfidation of small Pd NP is caused by the capping thiol molecules present not only on the surface but also in the bulk (Ramallo-López et al., 2007). Since it is an average technique, EXAFS alone cannot be used to characterize the size, structure or composition of NPs, without independent data of their distributions, especially in the case of multi-component NPs. The use of different complementary techniques and/or simulations is crucial for an accurate structural characterization of CS NPs. In situ XRD and EXAFS were used at the same time to monitor the oxidation process of the Co cores after thermal treatment at $800{ }^{\circ} \mathrm{C}$, either in air or under an inert atmosphere for $\mathrm{Co@} \mathrm{SiO}_{2} \mathrm{CS} \mathrm{NP}$ (Zhang et al., 2015). The findings allowed establishing that Co was oxidized in three steps no matter if air or $\mathrm{N}_{2}$ gas was employed during the annealing treatment. In another example, Leveneur et al. (2011) studied the nucleation and growth of Fe NPs in $\mathrm{SiO}_{2}$ by TEM, XPS and XANES. It was demonstrated that, using the ion implantation method, the entities initially resulted in the formation of dilute cationic $\mathrm{Fe}^{2+}$ species, while at higher dissolved iron concentrations, the formation of small metallic clusters was observed, which seed the particle growth during prolonged implantation or annealing. As already mentioned, XANES is a technique sensitive to coordination and bonding environment and it probes the unoccupied electronic states. Thus, this technique provides information 
about the crystal field (octahedral, square pyramidal or tetrahedral) that iron cations occupy. XANES complements the use of XPS, and it is particularly appropriate for nanostructures with complex compositions and various possible valence states for the iron.

Multicomponent NPs became an important class of materials due to their fascinating properties, electronic properties, synergistic effects, material-specific ensemble effects and ability to carry out multiple functions from the individual constituents. They are rich in capabilities and generate novel properties as a result of the interplay between their building blocks (Frenkel et al., 2012). These properties are directly affected by the details of the NP design - size, composition, homogeneity, structure, shape, surface morphology, reaction-driven restructuring, including the inorganic and organic constituent's effects, since the nature of capping ligands, solvent and nonsolvent are all contributing factors (Wang et al., 1997; Tao et al., 2008; Krylova et al., 2012). Understanding the modification of chemical composition during NP processing is also critical for the ability to design advanced, highly-performing heterostructures as multicomponent NPs. In particular, understanding the compositional uniformity and stability of CS NP is fundamental for further progress in the design of highly efficient catalysts, since the NP surface characteristics are critical for their performance (Stamenkovic et al., 2006). Additionally, regardless of the important progress in the synthesis of binary metal alloy NPs, there is still no clear understanding of the correlation between "bulk" composition and surface structure.

For all these reasons, one of the main interests is the precise and atomistic characterization of the nature of complex NPs, like multicomponent NPs or CS NPs; the measurement of geometric and compositional details with sufficient spatial resolution being one of the most challenging tasks. At present, without additional assistance of X-ray micro and nanoprobes (see next section), different experimental and complementary strategies are being devoted for such purposes, with the intrinsic limitations in each particular case. Thus, structural details rapidly diminish as the size of coherent scattering region decreases to a few $\mathrm{nm}$ in XRD, or advanced microscopy techniques (HRTEM, SEM, etc) are seriously limited to perform structural analysis out of the UHV, i.e. under controlled environmental conditions at elevated temperatures or moderate high pressure. Among available X-rays based techniques, many of them have shown indubitable contributions to these goals (Table 1). In effect, in addition to the more widely known EXAFS and XANES techniques, inner shell X-ray spectroscopies like X-ray emission spectroscopy (XES), high energy resolution fluorescence detection (HERFD), resonant inelastic X-ray scattering (RIXS), scattering (X-ray diffraction Pair Distribution Function (XRD/PDF) and small and wide angle X-ray scattering (SAXS/WAXS)) techniques have emerged as powerful research methods for investigations on inhomogeneous nanomaterials (Alayoglu et al., 2005; Singh et al., 2010; Glatzel et al., 2005).

Demanding efforts can be found in recent literature, combining X-ray based synchrotron techniques, in order to approach the problem of characterization of CS NP. Recently, M.D. Mizrahi at al. (2018) used small angle X-ray scattering, X-ray fluorescence and extended X-ray absorption fine structure spectroscopy to probe the structure of chemically synthesized CoPt3 NPs after ligand removal. They show that surface purification results in the considerable leaching of the Co atoms from the CoPt3 NPs, converting them into CoPt3@Pt core@shell structures with a thick Pt shell of half of nanometer. These results are also compatible with the improved catalytic activity of extensively purified NPs in octyne hydrogenation reaction found for the same samples.
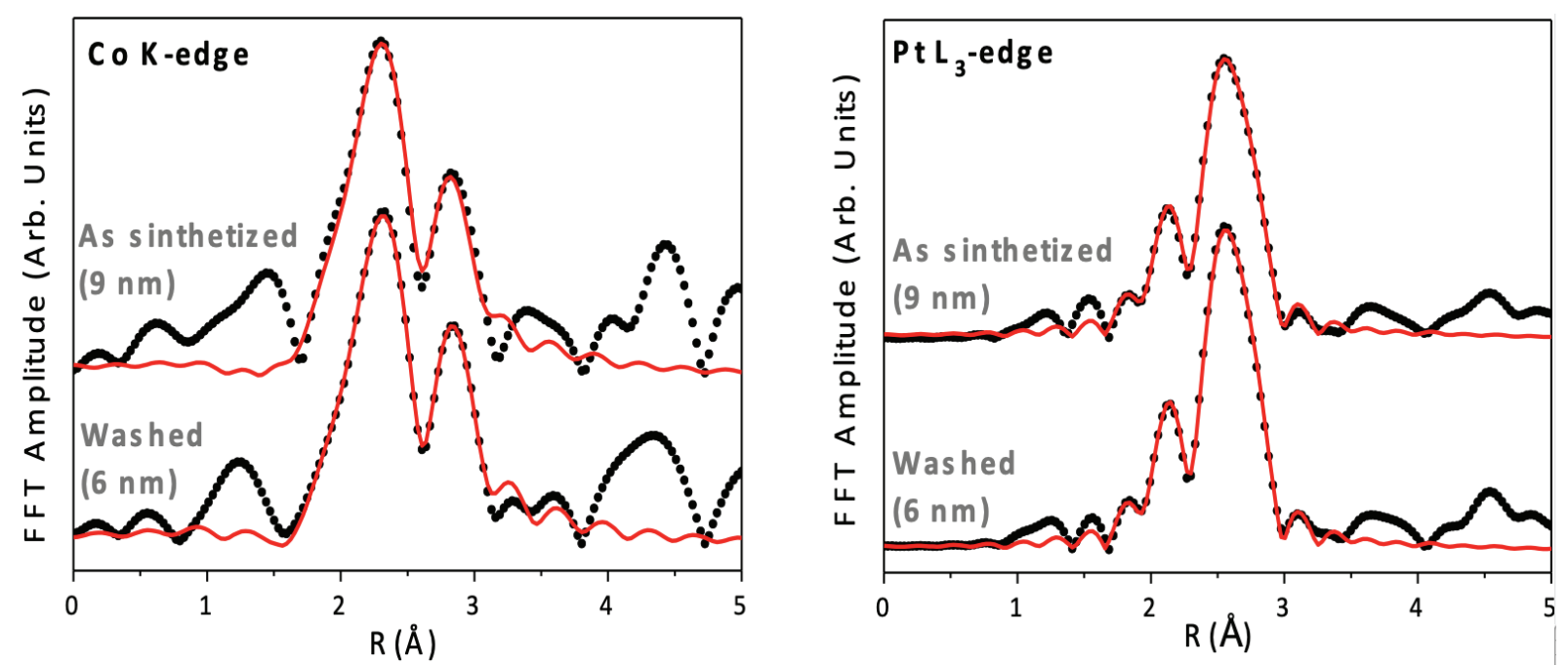

Figure 1. Experimental and fitted EXAFS Fourier Transform of fresh and washed NP samples at Co K and Pt L3-edges (left and right panels, respectively). Red lines correspond to the EXAFS fitting (see Table 2 for fitted parameters). 
NPs of alloys of Pt with transition metals present exceptional catalytic properties (Jiang et al., 2017; Li et al., 2015). The determination of the thin external shell is not evident in polycrystalline Pt alloyed with 3d-transition metals. However, the advanced catalytic properties of Pt-based alloy films were often attributed to the formation of Pt-skin (van der Vliet et al., 2012). The authors also demonstrate that drastic compositional and structural transformation occurs during the removal of native ligands via the solvent/non-solvent approach, leaching cobalt atoms in aqueous media. This kind of effect at surface of bimetallic NPs, experimenting complex transformations upon their exposure to different media, was reported previously. Thus, experimental studies on 10 to $12 \mathrm{~nm} \mathrm{Co-Pt} \mathrm{alloy} \mathrm{NPs} \mathrm{showed} \mathrm{the} \mathrm{migration} \mathrm{of} \mathrm{Co} \mathrm{atoms}$ and the formation of a strained epitaxial $\mathrm{CoO}$ film upon their high temperature exposure to oxygen, while in a reducing hydrogen atmosphere, Co atoms migrated back to the bulk, leaving a monolayer of platinum atoms on the surface (Xin et al., 2014).

In their work, Mizrahi et al. (2018) focus on understanding the effects of NP purification on surface composition and structure of bimetallic Pt-based alloy NPs synthetized in organic solvent. Many techniques are conjugated for that purpose: Transmission Electron Microscopy (TEM), Small Angle X-ray Scattering (SAXS), X-ray fluorescence (XRF) and Extended X-ray Adsorption Fine Structure spectroscopy (EXAFS), and all together provide a detailed analysis of the structure of chemically synthetized CoPt3 NPs after ligand removal. The purification process is applied on $9.3 \mathrm{~nm}$ CoPt3 NPs sample (in the following "as prepared" sample) to obtain $5.9 \mathrm{~nm}$ CoPt3 NPs sample (in the following "washed" sample) with the formation of $0.5 \mathrm{~nm}$ thick Pt shell. Thus, they demonstrate that the phase transfer of CoPt3 NPs and their prolonged exposure to water can result in the formation of thick Pt shell during extensive surface purification implemented for effective ligand exchanges and phase transfer.

Table 2. EXAFS Fitted parameters for both NPs as synthetized and washed samples at the K-Co edge (left) and L3-Pt edge (right). Parameters for CoPt and CoPt3 reference compounds are also included for comparison.

\begin{tabular}{|c|c|c|c|c|c|c|c|c|c|c|c|}
\hline \multicolumn{7}{|c|}{ K-Co } & \multicolumn{5}{|c|}{$\mathrm{L}_{3}-\mathrm{Pt}$} \\
\hline Sample & $\mathbf{N}_{\mathrm{CoPt}}$ & $\mathbf{R}_{\text {Co-Pt }}(\AA)$ & $\sigma_{C 0-P t}^{2}\left(\AA^{2}\right)$ & $\mathbf{N}_{\mathrm{CoCO}}$ & $\mathbf{R}_{\mathrm{Co}-\mathrm{Co}}(\AA)$ & $\mathbf{N}_{\text {PtPt }}$ & $\mathbf{R}_{\mathrm{Pt}-\mathrm{Pt}}(\AA)$ & $\sigma_{\text {Pt-Pt }}^{2}\left(\AA^{2}\right)$ & $\mathbf{N}_{\mathrm{PtC} 0}$ & $\mathbf{R}_{\mathrm{Pt}-\mathrm{C}_{0}}(\AA)$ & $\sigma_{\mathrm{Pt}-\mathrm{Co}}^{2}\left(\AA^{2}\right)$ \\
\hline As sinthetized & $11.4_{10}$ & $2.72_{3}$ & $0.009_{1}$ & -- & -- & $8.7_{4}$ & $2.74_{2}$ & $0.007_{1}$ & $1.9_{3}$ & $2.70_{2}$ & $0.009_{1}$ \\
\hline Washed & $10.6_{10}$ & $2.70_{3}$ & $0.008_{1}$ & -- & -- & $8.3_{4}$ & $2.73_{1}$ & $0.006_{1}$ & $1.7_{3}$ & $2.69_{1}$ & $0.007_{1}$ \\
\hline CoPt & 8 & 2.65 & & 4 & 2.69 & 4 & 2.69 & & 8 & 2.65 & \\
\hline $\mathrm{CoPt}_{3}$ & 12 & 2.71 & & 0 & -- & 8 & 2.71 & & 4 & 2.71 & \\
\hline
\end{tabular}

EXAFS was specially employed to analyze the local environment of Pt and Co separately in water-transferred NPs. Specifically, different phases or species segregation can be analyzed considering the fitted average coordination value $<\mathrm{N}>$, correlating it with the size of the cluster domain (Frenkel et al., 2011). Figure 1 shows the Fourier transforms of the EXAFS oscillations obtained at the Co-K and Pt-L3 edges, as well as the corresponding fitting curves for as-synthetized and washed samples. A model with two coordination shells with Co and Pt atoms as scatters in each shell is used to fit Pt-L3 EXAFS data, while only one coordination shell of Pt is enough to fit Co-K edge EXAFS data. EXAFS results at the Co K-edge revealed the Co-Pt interatomic distance of about 2.72(3) $\AA$, similar to 2.71, characteristic of the CoPt3 massive alloy. Only a Pt coordination shell was found around Co atoms, which is indicative of CoPt3 structure. Fitting of the EXAFS data obtained at Pt L3-edge also indicated that the Co-Pt interatomic distances are similar to those of CoPt3 alloy. Even though a contraction of Pt-Pt distances is expected (Nepijko et al., 1980) due to the enhancement of surface tension in NP, the interatomic Pt-Pt distances derived from Pt-L3 EXAFS fitting curve are greater than those in both CoPt3 and CoPt alloys. These EXAFS results can be explained by the coexistence of bimetallic and metallic phases. In effect, EXAFS fitting data indicate a higher number of the Pt atoms coordinated to other Pt atoms (8.7(4) vs. 8, characteristic to CoPt3 alloy) and lower number of Pt atoms coordinated to Co atoms (1.9(3) vs. 4, characteristic to CoPt3 alloy) (see Table 2). Thus, by double checking, EXAFS can probe, by both the interatomic distance and the average coordination numbers, the presence of two Pt-containing phases: metallic Pt and Pt-Co alloy, most likely CoPt3. Since NPs had spherical shape (probed by HRTEM and SAXS), it is reasonable to assume spherical CoPt3@Pt core@shell structure.

The effect of washing (purification) can also be inspected by XANES. In effect, the presence of a CoPt3 phase is also supported by Co K-edge XANES experiments (Figure 2). The positions of the characteristic features and the intensity of the white line in the Co K-edge XANES spectrum of extensively washed CoPt3 NPs are nearly the same as those of theCoPt3 bulk alloy reference (Figure 2) (the pre-edge feature at $7709 \mathrm{eV}$ and the bump at $7725 \mathrm{eV}$ that correspond to transitions from $1 \mathrm{~s}$ to $3 \mathrm{~d}$ and $1 \mathrm{~s}$ to $4 \mathrm{~d}$ orbitals in Co atoms, respectively). As regards as-synthesized $9.3 \mathrm{~nm}$ CoPt3 NPs, the CoPt3 XANES spectrum is clearly different, suggesting some differences, in average, in the local coordination of the Co atoms between as-synthetized and extensively purified CoPt3 NPs. The Co K-edge EXAFS study on as-synthetized 
CoPt3 NP indicates the presence of additional coordination shells for Co with Co-Co and Co-O contributions (not shown here), consistent with the presence of oxidized Co atoms at the surface of the as-synthetized NPs.

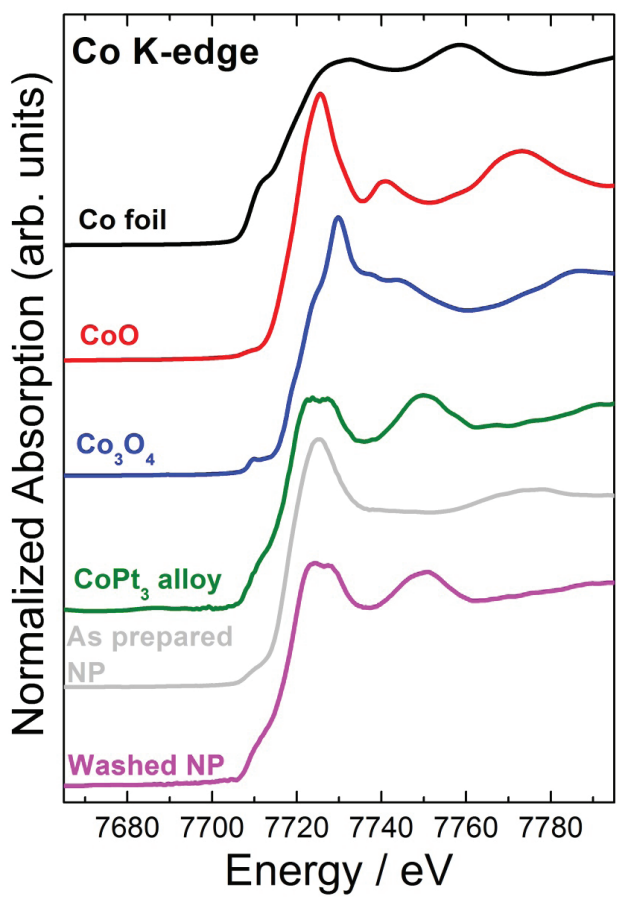

Figure 2. XANES spectra at the Co-K edge of as-prepared and washed samples compared to metallic Co foil, CoO, $\mathrm{Co} 3 \mathrm{O} 4$ and $\mathrm{CoPt} 3$ alloy reference compounds. Note the energy edge position of samples coincident with metallic Co foil reference and the similarity between the washed sample and the CoPt3 alloy reference compound.

Since the average coordination number $<\mathrm{N}>$ is directly associated with the geometry in the solid, sensitivity increasing as particle size decreases, the fitted $<\mathrm{N}>$ value from EXAFS experiments can be used to infer the size of the particle (assumed spherical). With this goal, we can calculate the theoretical average coordination numbers $\mathrm{N}_{\mathrm{Pt}-\mathrm{Pt}}$ and $\mathrm{N}_{\mathrm{Pt}-\mathrm{Co}}$ (Figure 3, red and blue curves, respectively) for CoPt3@Pt core@shells NPs with different CoPt3 core radii, keeping the external radius of NPs fixed to $4.65 \mathrm{~nm}$ as it follows from the TEM data. These findings will allow the estimation of the alloy core in water transferred NPs. In Figure 3, the horizontal green bars represent the average coordination numbers $\mathrm{N}_{\text {Pt-Pt }}$ and $\mathrm{N}_{\text {Pt- }}$ Co determined by the EXAFS experiments. Then, the area of intersection between the grey bars and the calculated curves (Figure 3, vertical bar) shows that the radius of the CoPt3 core was in the range between $3.8 \mathrm{~nm}$ and $4 \mathrm{~nm}$ (corresponding to a larger thickness of the Pt shell of about $0.75 \mathrm{~nm}$, estimated by the analysis of the concentration of the leached ions) (Krylova et al., 2010). Figure 4 shows the representation of the possible structure for as-prepared and washed NPs.
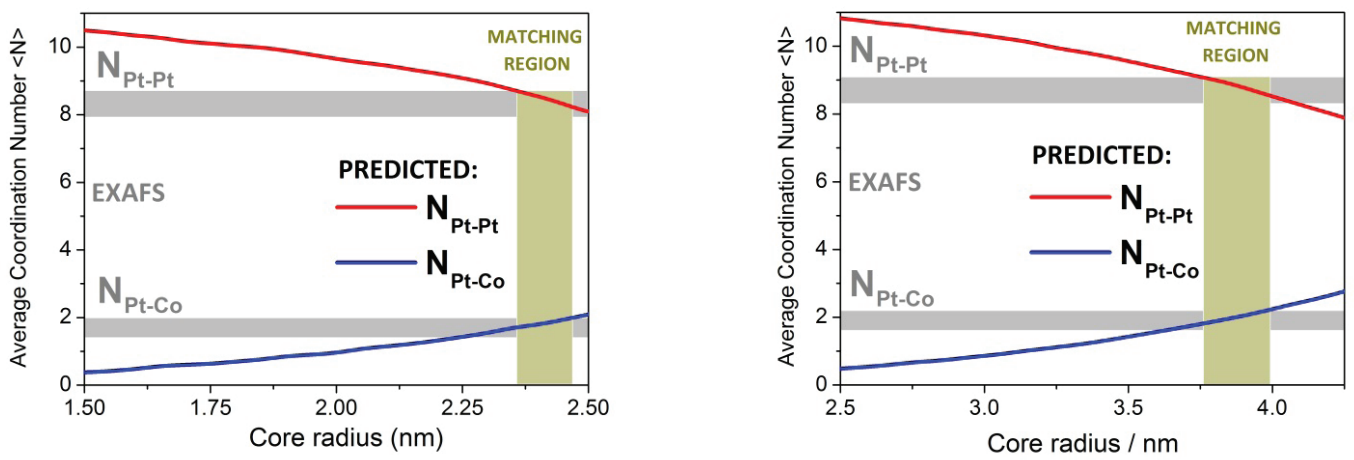

Figure 3. Calculated average coordination numbers, $\mathrm{N}_{\mathrm{Pt} \text {-Pt }}$ (red line) and $\mathrm{N}_{\mathrm{Pt} \text {-Co }}$ (blue line) as a function of CoPt3 core radius NP as prepared and washed (left and right panel respectively). The grey horizontal bars represent the average coordination numbers derived from the EXAFS fits. The vertical bar indicates range of the CoPt3 core radius NP that allows obtaining the coordination numbers matching the experimental data. 


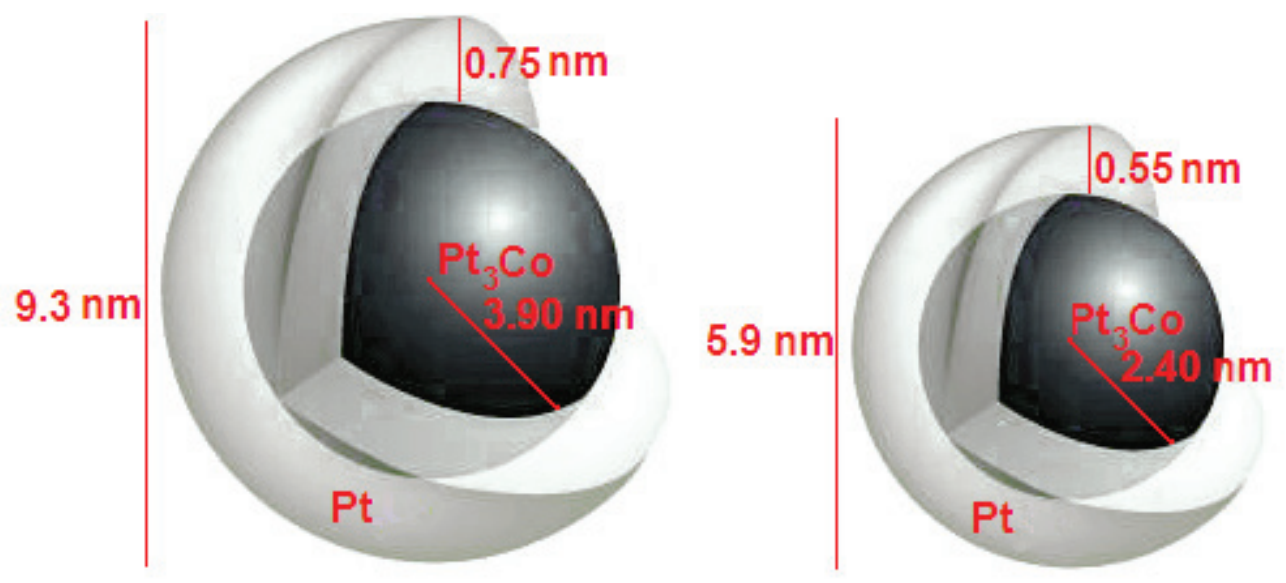

Figure 4. Representation of the proposed core@shell structure for as-synthetized and washed NPs (left and right respectively) derived from EXAFS fittings and XANES spectra, compatible with calculated averaged coordination number for determined structures.

XRF data allows confirming the derived estimation of the Pt-to-Co atoms ratio, based on the core@shell model with about $0.75 \mathrm{~nm}$ shell. The estimated value is 5.7(6), in good agreement with the ratio of 5.4 calculated based on the model. On the contrary, when the alternative configuration, such as Pt@CoPt3 and a random distribution of Pt and Co atoms, is calculated, a value of 4.8 is obtained, which does not match experimental florescence experiment results. These findings are also in agreement with EXAFS predictions, making the hypothesis even more robust.

An additional and direct experimental validation of the CoPt3@Pt core@shell model was performed by SAXS on the same samples under the same conditions (in water solution at room temperature). The corresponding fitting with a core of CoPt3 alloy and Pt atoms shell model is shown in Figure 5. The mean core radius and shell thickness values obtained by SAXS fittings are in good agreement with EXAFS results. The advantage of SAXS with respect to TEM determinations is not only the fact the experiments can be performed on liquid samples, but also that SAXS provides information averaged over a large volume of NPs. This is much more representative of the general state of the sample, while TEM is limited to the analysis of hundreds of NPs.
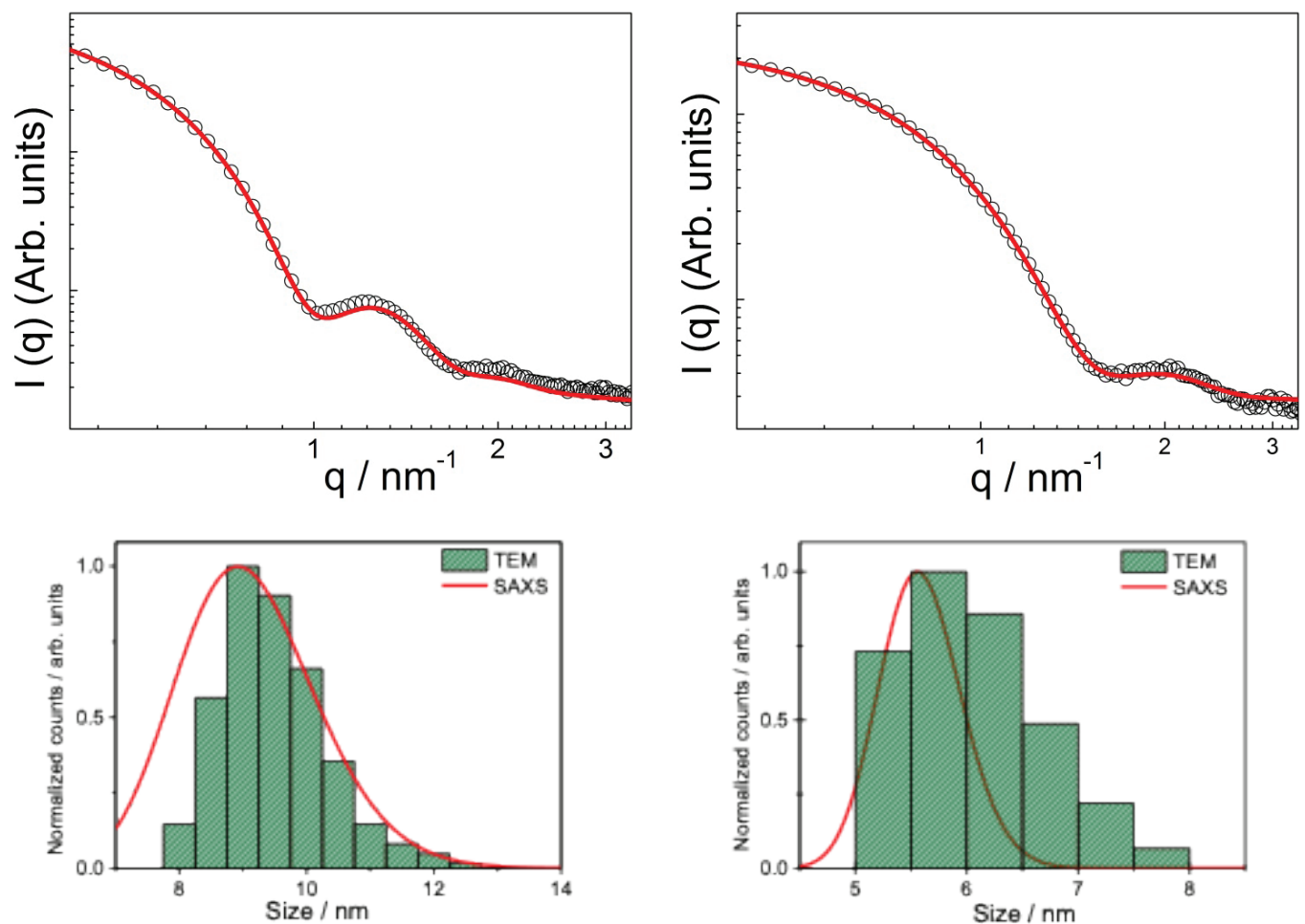

Figure 5. Experimental SAXS data (intensity vs. wave vector q - open symbols) for as-prepared and washed NPs (upper left and right panels, respectively) and the fitting curve obtained based on a spherical core/shell model. The size distributions reconstructed from fitting curve were overlapped with size distribution data obtained based on TEM data (lower panels). 
In conclusion, X-ray-based techniques EXAFS, XANES, XRF and SAXS consistently support the same model for the core@shell structure of water transferred NPs. Based on this, the authors suggest that cobalt atoms leached only from the surface of CoPt3 NPs based on the analysis of the concentrations of the leached Co $(+2)$ ions to the water by zero explains the suppression of CoPt3@Au dumbbells formation when extensively washed alloy NPs were used as seeds (Krylova et al., 2012). These findings, summarized in Table 3, also contribute to enlighten the control mechanism, by the solvent/ non-solvent approach, to regulate the properties of multicomponent, transition metal based NPs for wanted applications.

Table 3. Comparative summary indicating the contribution from each X-ray technique for the characterization of core@shell NPs.

\begin{tabular}{|l|l|l|}
\multicolumn{1}{|c|}{ Technique } & \multicolumn{1}{|c|}{ Data/Parameters } & \multicolumn{1}{c|}{ Information provided } \\
\hline EXAFS & $\begin{array}{l}\text { - Average coordination number } \\
\text { - Interatomic distances Pt-Pt, Pt-Co, Co-Pt and } \\
\text { Co-Co }\end{array}$ & $\begin{array}{l}\text { - Pt and Co-species, core@shell model } \\
\text { - Pt and Co species }\end{array}$ \\
\hline XANES & $\begin{array}{l}\text { - Absorption spectrum } \\
\text { - Energy edge position }\end{array}$ & $\begin{array}{l}\text { - Chemical speciation } \\
\text { - Average oxidation state for Co and Pt }\end{array}$ \\
\hline XR Fluorescence & $\begin{array}{l}\text { - Intensity of Co-K } \\
\text { lines (not shown in this work) }\end{array}$ & $\begin{array}{l}\text { - Co/Pt relative atomic concentration } \\
\text { - Pt and Co stoichiometry of species }\end{array}$ \\
\hline SAXS & $\begin{array}{l}\text { - Radii of gyration (considering the whole } \\
\text { particle) }\end{array}$ & $\begin{array}{l}\text { - Size distribution, shape, core@shell model, } \\
\text { core radius, shell thickness }\end{array}$ \\
\hline
\end{tabular}

\section{X-rays micro and nanoprobes: background, technical aspects and opportunities in nanoscience}

\section{Characteristics of X-rays micro and nanoprobes}

X-rays micro and nanoprobes (XMNP) represent an unbeatable opportunity for pioneering research, both for basic investigation and in close relation to practical industrial applications. The scientific projects planned to be performed at these types of beamlines at synchrotron laboratories cover different fields, where spatial resolution and chemistry (or electronics) play a fundamental role.
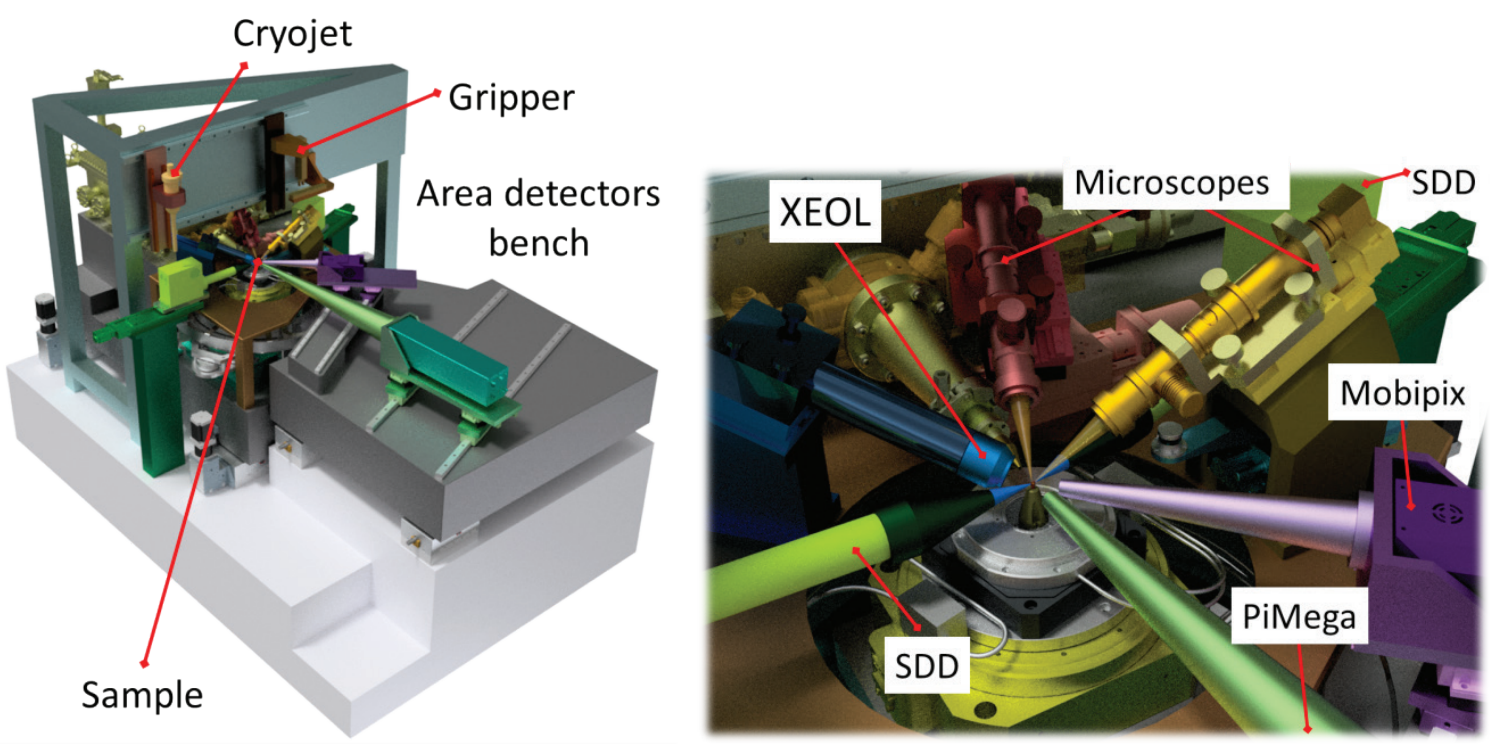

Figure 6. Scanning probe TARUMÃ at CARNAUBA nano and microprobe beamline. SIRIUS synchrotron laboratory in Campinas, Brazil. (Figure by courtesy of H. Tolentino, SIRIUS, Campinas, Brazil (H.C.N. Tolentino, R.R. Geraldes, 2019).

At these new XMNP end-stations, it is feasible to find opportunities for X-ray fluorescence (XRF), X-ray absorption fine structures (XAFS), and X-ray excited optical luminescence (XEOL). The primary enabling experimental technology 
for focusing X-rays to such small spots has been the development of X-ray optics. Their applications, however, have been limited by the trade-off between useful flux and resolution. In general, to satisfy the diverse requirements of various X-ray techniques, several design criteria are set for modern XMNP beamlines: (1) minimum number of beamline optical elements to preserve source coherence; (2) nano-focusing optics with a diffraction-limited spot; (3) high throughput optics; (4) achromatic optics; (5) large working distance; and (6) minimum mechanical and ground instabilities. The technical qualities of different beamlines for XNMP are outlined in Table 4.

Representative scientific cases that can be specially afforded by XMNP beamlines cover, in general, a diverse field of research, such as material science, MEMS/NEMS device characterization, cultural heritage, in vivo and in vitro bio-materials and cells, geology, etc. In the following section, specific examples are reported, in particular in the field of material science characterization.

Table 4. Characteristic parameters of recent and planned and constructed X-ray nano-probe beamlines.

\begin{tabular}{|c|c|c|c|c|c|}
\hline Beamline/Syncrotron & $\begin{array}{l}\text { Energy } \\
\text { range } \\
{[\mathrm{keV}]}\end{array}$ & $\begin{array}{l}\text { Energy reso- } \\
\text { lution } \\
{[\Delta \mathrm{E} / \mathrm{E}]}\end{array}$ & $\begin{array}{l}\text { Beam size } \\
{[\mathrm{nm}]} \\
\text { (focal point) }\end{array}$ & $\begin{array}{l}\text { Photon flux } \\
\text { [photons/s] }\end{array}$ & $\begin{array}{l}\text { Emittance } \\
\text { [nm·rad] } \\
\text { (synchrotron } \\
\text { ring) }\end{array}$ \\
\hline $\begin{array}{l}\text { XNP, Taiwan Photon } \\
\text { Source (Hsinchu, Taiwan) } \\
\text { (Yin et al., 2016) }\end{array}$ & $4-15$ & $\begin{array}{l}\leq 1.5 \times 10^{-4} \\
\operatorname{Si}(111)\end{array}$ & $\begin{array}{l}40 \\
\text { (@) } 10 \mathrm{keV})\end{array}$ & $1-10 \times 10^{10}$ & $\begin{array}{l}1.6 \\
\text { (a) } 3 \mathrm{GeV}\end{array}$ \\
\hline $\begin{array}{l}\text { Carnauba, SIRIUS Cam- } \\
\text { pinas, Brazil } \\
\text { (Tolentino et al., 2017; To- } \\
\text { lentino et al., 2019) }\end{array}$ & $2.05-15$ & $1.0 \times 10^{-4}$ & $\begin{array}{l}\text { TARUMÃ } \\
\text { (sub-Microprobe) } \\
500-100 \\
\text { SAPOTI (Na- } \\
\text { noprobe) } \\
120-30\end{array}$ & $\begin{array}{l}3.5 \times 10^{12} \\
(2 \mathrm{keV}) \\
2.5 \times 10^{12} \\
(4 \mathrm{keV}) \\
1 \times 10^{12} \\
(10 \mathrm{keV})\end{array}$ & $\begin{array}{l}0.25 \\
\text { (a) } 3 \mathrm{GeV}\end{array}$ \\
\hline $\begin{array}{l}\text { ID16B, ESRF } \\
\text { Grenoble, France. } \\
\text { (Martínez et al., 2016) }\end{array}$ & $6-65$ & $\begin{array}{l}10^{-2}(\mathrm{PM} \#) \\
10^{-4}(\mathrm{MM} \#)\end{array}$ & $\begin{array}{l}50 \times 50(\mathrm{PM}) \\
80 \times 100(\mathrm{MM})\end{array}$ & $\begin{array}{l}10^{11}-10^{12}(\mathrm{PM}) \\
10^{8}-10^{9}(\mathrm{MM})\end{array}$ & $\begin{array}{l}3.8 \\
\text { (a) } 6 \mathrm{GeV}\end{array}$ \\
\hline $\begin{array}{l}\text { HXN, NSLS II } \\
\text { Brookhaven, USA. (Naza- } \\
\text { retski et al., 2017) }\end{array}$ & $6-25$ & $1.3 \times 10^{-4}$ & $\begin{array}{l}10 \times 10\left(\mathrm{MLL}^{*}\right) \\
30 \times 30\left(\mathrm{FZP}^{* *}\right)\end{array}$ & $\begin{array}{l}>5 \times 10^{8} \\
(10 \mathrm{keV})\end{array}$ & $\begin{array}{l}<0.5(\mathrm{H}) \\
\times 0.008(\mathrm{~V}) \\
@ 3 \mathrm{GeV}\end{array}$ \\
\hline
\end{tabular}

\# pink mode, \#\# monochromatic mode, * Multilayer Laue Lens, ** Zone Plate Focussing

\section{Present and future of X-ray probes in nanoscience}

Third-generation synchrotrons start the revolution in scientific instrumentations over the past decade. In particular, the development of advanced imaging micro and nanoprobes constitutes a technology breakthrough in the field of x-ray optics (De Andrade et al., 2011; Nazaretski et al., 2015; Shapiro et al., 2014; Winarski et al., 2012). Different unique characteristics of X-rays, such as resolution, sensitivity and speed, and their ability to penetrate matter, constitute an important opportunity for the future for scientific research in nanoscience. The varying ability of different probes compared with $\mathrm{X}$-rays still needs to be bridged in terms of spatial resolution. This is a very challenging field where the spatial resolution limit is pushed constantly. However, the ability of spatial resolution must be weighed integrally comparing different probes, considering pros and cons, for a realistic assessment. In effect, among photons, different probes can be considered to obtain space-resolved structural or compositional information, including photons obtained from laser sources, electrons, neutrons, and ions (Mino et al., 2018). Even if laser and x-ray microprobes have similar characteristics (they are both based on photons), the main differences between them are their wavelength range (while laser microprobes operate within a wavelength range of a few microns to around $200 \mathrm{~nm}$, x rays work within a 10-0.01 nm range) and the much higher coherence of laser beams compared to synchrotron x-ray sources. Having a lot more versions of different methodologies to approach different topics, the comparison between electron and X-rays microprobes deserves more comments 
and comparisons: XRD versus electron diffraction, XAS versus electron energy loss spectroscopy (EELS), XRF versus energy-dispersive $\mathrm{X}$-ray (EDX) spectrometry, $\mathrm{x}$-ray emission spectroscopy (XES) versus electron probe microanalysis (EPMA). Our aim in this section is not focusing on an in-depth discussion of all these comparisons, but mentioning the ones that can be applied to relevant aspects of nanomaterials characterization.

It is already well known and already spread in most of the laboratories that a single electron microscope offers multiple characterization techniques, combined with an outstanding spatial resolution. Despite the well-known capabilities of electron microscopes based-techniques, there are many disadvantages that limit their use for the characterization of several samples. In particular, sample preparation could be a strong limitation in electron microscope studies, which can be challenging and could even modify the intrinsic properties of the samples to be studied - the requirement of very thin samples $(\leq 100 \mathrm{~nm})$, sometimes the need to prepare sample using sophisticated procedures such as focused ion beam (FIB) setups. As the reverse of the surface selectivity of electron probe methods, there is a strong limitation for a bulk characterization or for the analysis of buried or embedded volume portions. Regarding sample damage during experiments, the intense electron beams or higher energies required to increase penetration depth can result in significant radiation damage, mainly because electrons are capable of transferring relatively large momenta to the sample (García de Abajo et al., 2010). Another critic disadvantage for techniques based on electron probes is the need to operate under high vacuum, with the obvious limitations for reproducing different in situ environment setups for many types of research topics. As regards the known chemical selectivity of X-rays, unless XRF or Auger spectroscopies are used as probes, electron microscopes present an important drawback compared to $\mathrm{x}$ rays. On the other hand, ion probes have the significant particularity, as opposed to photons or electrons probes, that they deposit extremely localized densities of energy to the target in a very short time (from the kinetic energy of ions) (Ziegler et al., 1985; Avasthi et al., 2011). However, the size of the spot in this case can be a very small region of about $10 \mathrm{~nm}$ with advanced focusing and/or collimation (Yao et al., 2015). Finally, neutron probes, extensively employed, include many advantages compared to the rest of probes, and they can complement the advantages of X-rays probes. Among them, we can distinguish sensitivity to hydrogen (Neumann, 2006), isotope specificity, and magnetic interaction (Fitzsimmons et al., 2004). Finally, the strong penetration depth makes it ideal for bulk probes. Nevertheless, a major obstacle in the (sub)micrometric characterization of materials with neutrons, besides the technical limitation in manufacturing the focusing devices, currently located in the range of fraction of mm, is the relatively low flux provided by present neutron sources. To obtain a similar brightness of $\mathrm{x}$ rays produced by third- and fourth-generation synchrotron, for neutron sources it necessary to increase the production of neutrons about 2 orders of magnitude.

Let us consider the X-ray-based methods and their opportunities with the micro and nanoprobes versions in more detail. X-ray probes can offer outstanding possibilities for nondestructive characterization at the (sub)micrometric scale, overcoming many limitations already described by other probes like ions, neutrons or electrons. In general, their advantages include the availability of several, often complementary, physical process or magnitudes (e.g., absorption, emission, scattering, diffraction, phase contrast, and polarization), simple sample preparation procedures, and due to the long mean free path of energetic photons, the possibility to perform experiments at in situ or operando conditions. In effect, energetic $\mathrm{x}$ rays (hard-X-rays) have low cross sections for their interaction with matter, allowing nondestructive characterization experiments, applicable at pressures around atmospheric conditions, liquid media like water, and any other non-strong absorbing or dispersive environments. These low cross-section values also involve high penetration depths through volume matter, offering the opportunity of $3 \mathrm{D}$ analysis. At this time, the available or programmed $4^{\text {th }}$ generation synchrotrons, with high brilliance and low emittance beams facilitates high density of data, with reasonable signal-to-noise ratio, even at the (sub)micrometric scale with relatively short acquisition times.

Different micro and nano-spectroscopic techniques are currently in constant development. Thus, we can find x-ray absorption for micro- and nano-XAS (XANES) (Cotte et al., 2011), EXAFS (Martínez-Criado et al., 2014) and polarization-dependent methods, e.g. micro- and nano-XMCD (Suzuki, 2014) (x-ray magnetic circular dichroism), or very advanced spectroscopic approaches such as RIXS (resonant inelastic X-ray scattering). Still more established microspectroscopic techniques are micro-XRF (Provis et al., 2009), micro-XEOL (Villanova et al., 2012) x-ray-excited optical luminescence and micro(nano)-XPS (Kolmakov et al., 2008) (x-ray photoelectron spectroscopy).

\section{Diversity of scientific cases with X-ray micro and nano-probes studies}

Typically, X-ray based synchrotron techniques are presented as ensemble averages, and sometimes this characteristic is complementary to traditional microscopic ones, which probe objects individually. Currently, due to recent advances in X-ray sources, X-ray optics and X-ray methods, XMNP emerges as unprecedented and possible tools to image crystalline structure distributions in materials science. Thus, nanobeams combined with diffraction have made possible imaging parameters in individual nanocrystals, and coherent nanobeams offer the opportunity to image nanomaterials in three dimensions with a resolution far smaller than that of the traditional focused beam size. Thus, the traditional requirement about sample homogeneity for X-ray absorption techniques can be omitted nowadays. This "old" precept can be abandoned since the allowed spatial resolution allows studying homogeneous regions in highly heterogeneous samples. 
Combining of advanced X-ray characterization tools with higher-resolution imaging techniques facilitates further breakthroughs. In the field of technological applications, the synchrotron microanalysis technique applied to potential photovoltaic materials is one of the examples with high impact. New characterization tools allow developing advanced materials for solar cells (Villanova et al., 2012), unraveling the structure-property relationship thanks to the large penetration depths, high throughput and good tunability (energy, spot size, photon flux, polarization, etc.). One important aspect is the possibility of operando and in-situ studies - hard X-ray beam intensities allow simultaneous signal acquisition and analysis of buried structures and mechanism process studies under realistic thermodynamic conditions. Different methodologies already demonstrated their efficiency on solar-cells characterization - X-ray diffraction (XRD) and small-angle X-ray scattering (SAXS) techniques have provided structural information at the atomic and mesoscopic scales (Schorr, 2011; Chiu et al., 2008), and X-ray absorption spectroscopy (XAS) has delivered information on local phenomena helping identify degradation and failure mechanisms (Buonassisi et al., 2005). X-ray microprobe beams can also be applied for material characterization of MEMS/NEMS devices (Bleuet et al., 2009). In effect, the structure of microsensors or micro-actuators must be nondestructively evaluated. TEM/SEM are frequently used because of their better resolution compared to X-ray microscopy, X-ray microprobe being better for probing deeper regions into the sample. Additional advantages are found for X-ray imaging due to the possibility of working under non-high vacuum conditions, the absence of special requirements for sample conductivity, and less tedious sample preparation procedure, especially for non-conductive samples. Innovative applications of synchrotron X-ray nano-probes in the field of cultural heritage can also be found. This modern, non-destructive application of nano-beams can produce high-spatial-resolution information on fragmented samples in historical or model artworks and constitutes a unique opportunity to revisit those fields of research (Cotte et al., 2018). Different techniques, such as nano-X-ray fluorescence, can be applied to probe elements. Nano-X-ray diffraction is useful to identify crystalline phases, and nano X-ray absorption spectroscopy is a sensitive tool for speciation. More challenging, computed tomography-based techniques can provide additional information about the morphology and porosity of materials.

A special chapter can be devoted to bio-metals imaging and speciation in cells using proton and synchrotron radiation $\mathrm{X}$-ray microspectroscopy. XMNP allows chemical element analysis in subcellular compartments. Indeed, the imaging and quantification of trace elements in single cells can be obtained using particle-induced X-ray emission (PIXE). The combination of PIXE with backscattering spectrometry and scanning transmission ion microscopy provides a high accuracy in elemental quantification of cellular organelles. On the other hand, synchrotron radiation X-ray fluorescence provides chemical element imaging with less than $100 \mathrm{~nm}$ spatial resolution. High resolution multi-element analysis techniques are especially relevant because they allow simultaneous mapping of essential elements. In this context, two promising techniques for characterizing the sub-cellular distribution of metallodrugs are secondary ion mass spectrometry (SIMS) and synchrotron-based X-ray fluorescence (SXRF). In vivo and in vitro studies are possible by micro-SXRF studies (for instance, for anticancer metal compounds), determining metal distribution inside cells for many elements at the same time (Morrison et al., 2014; Wu, L.E. et al., 2016). Additionally, synchrotron radiation offers the unique capability of spatially resolved chemical speciation using micro-X-ray absorption spectroscopy (Sanchez-Cano et al., 2019).

\section{Micro and nanoprobes for nanomaterials characterization}

Clearly, nanoscience and nanotechnology are two of the research and technology fields that benefited the most - from advanced electronics to energy harvesting, energy storage and catalysis. A wide range of actions became a reality - creation and manipulation of structural features such as defects, connections with other structural and size effects, metastable structural phases, precisely strained materials, and precisely designed nanoscale configurations of magnetic and electronic order (Hofmann et al., 2017; Ice et al., 2011; Holt et al., 2013). Constant and challenging issues in the field of nanoscience find today a new opportunity to be solved - understanding macroscopic materials through their nanoscale structural properties, the ability to characterize the resulting structure with high structural precision and in an appropriate timescale. In summary, the general study of the effects of structure, environment, and time-evolution of defects, interfaces, and domains at nanometric scale on material properties can be boarded by new micro- and nanoprobe-based techniques.

X-ray diffraction in isolated nanocrystals reveals details of their specific mechanical and chemical properties in comparison to their bulk counterparts. The sensitivity of the technique to interatomic distances, combined with the spatial resolution (between 10 to $1000 \mathrm{~nm}$ ), both reveals and corroborates the presence of microstructural heterogeneities that, from traditional X-ray diffraction experiments, were previously only described by averaged information. Thus, this emerging approach allows to reveal the mechanisms through which the enhanced properties of nanomaterials emerge in great detail in a vast number of hard condensed matter systems. It covers basic and applied areas, such as ceramics or metals, where their macroscopic mechanical properties are defined at the mesoscale by the interaction of defects and crystallites. Magnetic or multiferroic materials are additional examples where domain structures can now be resolved, up to technological fields ranging from catalysis to batteries, where the local influence of dislocations on damage mechanisms, during charge and discharge cycles of Li-ion batteries, can be imaged with coherent X-ray nanobeams (Ulvestad et al., 2015) or discern- 
ing strain fields in semiconductor devices during process at relevant temperatures (Vianne et al., 2015). More specifically, the information concerning the local structures and electronic structures surrounding the dopants and constituent atoms in nanostructures is essential to understand the fundamental mechanism leading the favorable properties. Indeed, it has been demonstrated that nanocrystals of semiconductors with different shapes present widened bandgaps, ultra-fast transitions, and photoluminescence efficiencies due to the quantum confinement effects, which can be explained based on their unique physical properties (Yan et al., 2015).

The cocktail of chemical selective techniques like nano-EXAFS, nano-XANES and nano-XEOL, using focused X-ray beam on isolated nanostructured semiconductors, can be used to distinguish different regions, like surfactants or substrates. Moreover, in the case of nanoscaled functional materials, it becomes essential to correlate structural and optical properties by applying different techniques sensitive to the structure and chemistry to the same nano-object (where the use of non-destructive and contact-less techniques is convenient). The traditional techniques employed for the study of the core@shell NPs, up to this moment, had been the high resolution scanning transmission electron microscopy (HR-STEM) (Koester et al., 2011), with the already described limitations or constraints.

\section{Micro and nanoprobes for inhomogeneous nanomaterials characterizations}

Recently, Secco et al. (2019) performed a detailed study of GaN/InGaN nanowire (NW) heterostructures. Individual NWs were dispersed on $200 \mathrm{~nm}$-thick SiN windows for measurement at the nanoimaging station ID16B of the European Synchrotron Radiation Facility (ESRF, Grenoble, France). The authors claimed that such InGa-based nanowires forming core@shell structures, typically present inhomogeneities in their elemental composition, defect concentration, and strain fields at the nanoscale. Being their characterization very complex and costly by conventional techniques, the synchrotron $\mathrm{X}$-ray nanoprobe emerges as an alternative multi technique approach. Thus, it is possible to follow the elemental composition and distribution by X-ray fluorescence (XRF), strain fields and crystal quality by X-ray diffraction (XRD), and local order effects and elemental segregation by X-ray absorption near edge structure (XANES) spectroscopy.

The distribution of Ga and In in the NWs could be elucidated from the XRF intensity maps (Figure 7), showing that $\mathrm{Ga}$ is homogeneously distributed along the NW axis. Complementarily, the intensity of In shows an increment from the bottom to the top end of the NW (the higher intensity of In at the top of the NW does not correspond to the formation of polar MQWs, which is demonstrated by EDS). Au and Ag maps (see Figure 8) lead to the conclusion that no catalyst atoms are disseminated in the NWs. Additional evidences are achieved from longitudinal and radial scans of the XRF (see Figure 7). These profiles confirm that there is an InGaN shell structure with an increasing concentration of In towards the top of the NW. Ga atoms, on the other hand, are more evenly distributed. 

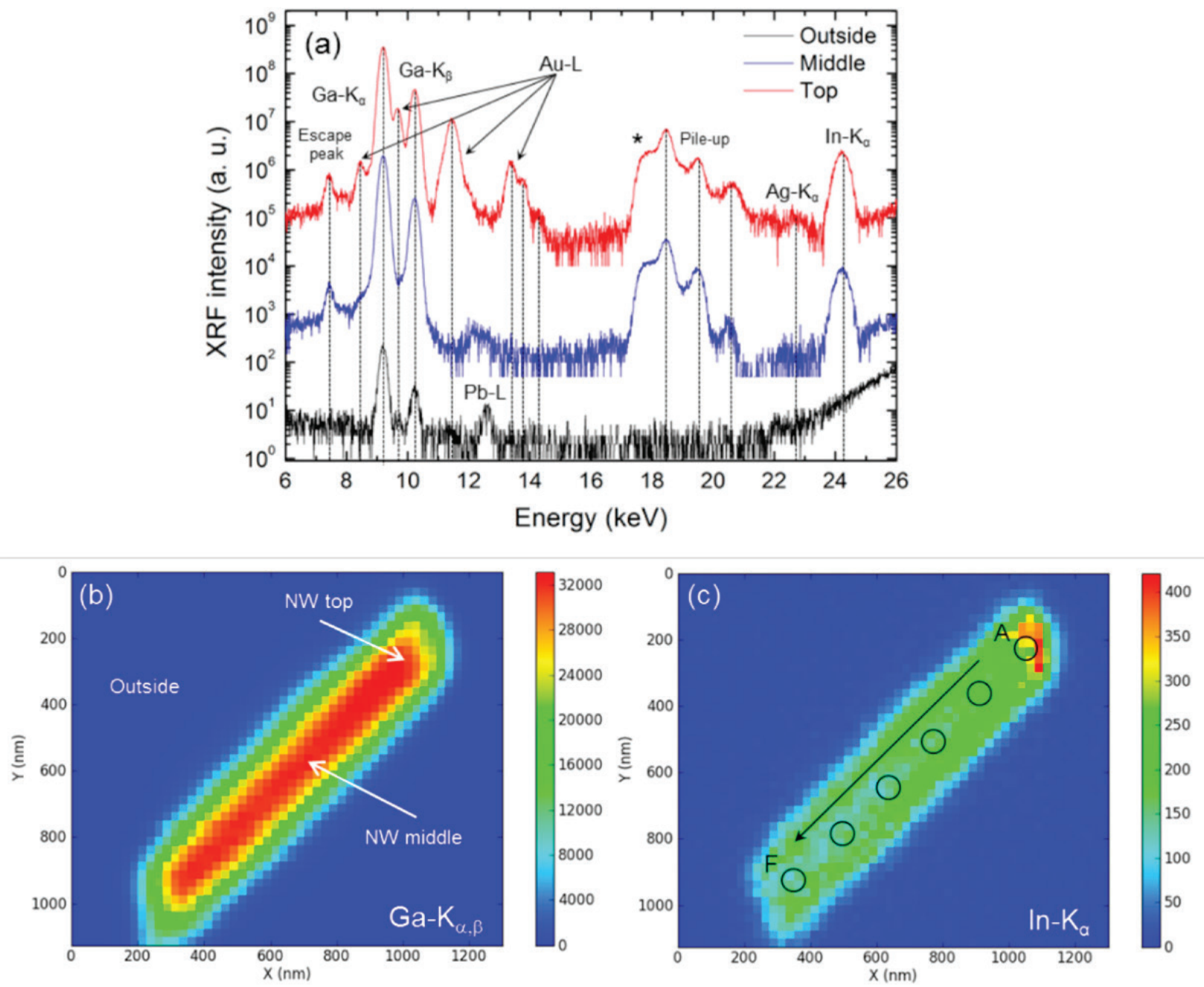

Figure 7. (a) Averaged X-ray fluorescence (XRF) spectra from locations at the top (red) and middle (blue) of the NW, and at an outside region (black). The labels indicate the elements associated with each peak identified with PYMCA. The asterisk represents an artifact coming from the measurements. The XRF intensity color maps of Ga and In in the scanned area are shown in (b) and (c), respectively. Red (blue) color corresponds to high (low) fluorescence intensity (scale in counts). The black circles in (c) indicate the regions of NW along its axis in which In concentration has been estimated (Figure published with permission of Secco et al. (2019).
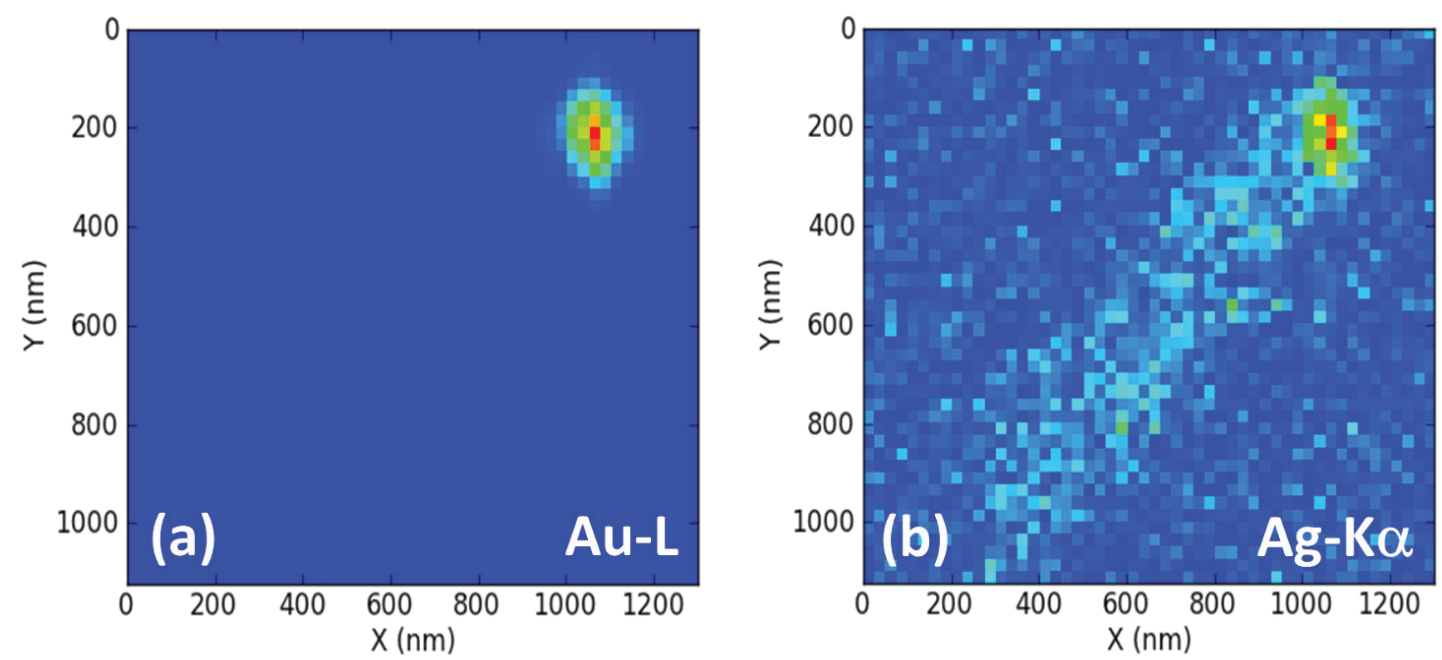

Figure 8. XRF colour map distribution of (a) Au and (b) Ag along a representative NW. The colour scale represents the $\mathrm{XRF}$ intensity in photon counts and ranges from 0 to 9000 (for $\mathrm{Au}$ ) and from 0 to 25 (for Ag) (Figure published with permission of Secco et al. (2019). 
The crystal phase and lattice parameters of different regions across single NWs can be investigated by nano-XRD. The short distance separating the CCD and the sample allowed measuring three diffraction peaks simultaneously: (104), (210), and (211) reflections of unstrained wurtzite GaN, which were only detected by analyzing the XRD signal of the whole $\mathrm{NW}$, suggesting that the core-shell InGaN MQWs are completely lattice matched to the strain-free GaN core. This is also confirmed by the absence of a distinctive XRD peak coming from core-shell MQWs, which are completely strained (compressed along the $a$ and $c$ directions and expanded along the m-direction). Additionally, XRD reflections allow calculating the interplanar distance, dhkl, using the general relation between interplanar distances (d), Miller indices (hkl), and lattice parameters $(a$ and $c$ ) in the wurtzite structure. Thus, lattice parameters $a$ and $c$ in the studied NWs can be measured, their evolution along the $z$-axis of the NW showing no evolution in $a$ and a non-monotonous increase in $c$ towards the NW top. The studied area starts at the middle and goes up the top of the NW (no signal was registered between the bottom and the middle of the NW due to the loss of the Bragg condition at imperfections on the NW).

Complementary to XRD measurements, which probe the long-range structural order, XANES experiments were performed for studying the local arrangement around Ga atoms in NWs. Figure 9 shows typical XANES spectra for a single NW using perpendicular and parallel polarization in relation to the NW axis, X-ray beam. For comparative purposes, a high-quality $c$-oriented $\mathrm{GaN}$ reference layer was also measured with polarized X-rays in relation to the wurtzite $c$-axis. This comparison allows concluding, since the spectra of the NW matches that of the reference, that the NW has a wurtzite structure and there is no mixture of phases. Indeed, the axis of the NW corresponds to the c-axis of the wurtzite structure.

This characterization is an illustrative example where the combination of XRF and EDS gives a reliable picture of the detailed structural characterization, despite the complexity of the NWs nanostructures, because of their inhomogeneities in composition and strain at a nanometer length scale. Similarly, XANES and XRD probe local and long-range order in such heterostructures - determinations of the In concentration in the QWs allows strain state calculations from XRD spectra. Finally, In content fluctuations can explain the variations in the emission energy and the broadening of emission peaks photoluminescence spectra in individual NWs, which can be interpreted and modeled theoretically.
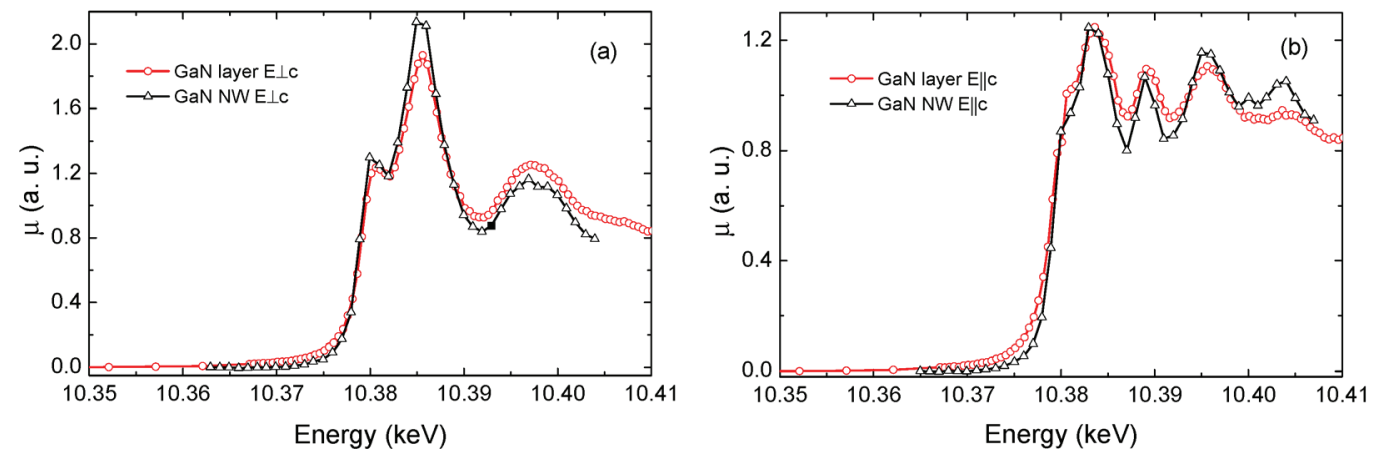

Figure 9. Comparison between X-ray absorption near edge structure (XANES) spectra of a high quality reference GaN layer (red circles) and those of the NW (black triangles) for different X-ray beam incident angles: polarization (a) perpendicular and (b) parallel to the wurtzite $c$-axis (Figure published with permission of Secco et al., 2019).

\section{Conclusions}

We listed a series of experimental methodologies based on X-ray probes that offer unique possibilities for nondestructive characterization, overcoming many limitations present in other probes such as ions, neutrons or electrons. The different techniques are based on complementary physical process (e.g., absorption, emission, scattering, diffraction, phase contrast, and polarization), which are compatible with feasible sample preparation and the possibility, in general, to perform experiments at in situ or operando conditions.

The conventional version of the techniques mentioned, accessible at third generation synchrotron sources, requires the conjunction of several (and different) experiments to arrive at a convincing conclusion. Due to the advent of the new fourth generation of synchrotron sources, with very low emittance, micro and nanoprobes end-stations are the perfect tools for studies that can be more directly reached by only single shot, simultaneously analyzing the emerging probes at very high spatial resolution. The chosen examples of core@shell nanostructures observed with and without spatial resolution illustrate this.

The application of micro- and nanoprobes in the general field of nanoscience research is really auspicious. The X-ray micro- and nano-analytical techniques are quite unique tools for corroborating structures, chemical species, electronics, 
magnetism and any other fundamental characteristic. Systems under study can vary from agglomerated atoms or molecules in confined regions to intracellular targets or very inhomogeneous structures, at different thermodynamic conditions, extending the limits of scientific experimentation.

\section{Acknowledgments}

The author acknowledges the permanent support and assistance from LNLS (Laboratório Nacional de Luz Síncrotron, Campinas, Brazil) for its contribution to the development of scientific research in the region. The devoted attitude for overnight experimentation of the SUNSET group of INIFTA in La Plata, Argentina, is also acknowledged. CONICET and ANPCyT partially supported research using synchrotron laboratories.

\section{References}

[1] M. Acebrón, F.C. Herrera, M. Mizrahi, C. Navío, R. Bernardo-Gavito, D. Granados, F.G. Requejo and B.H. Juarez. Inorganically coated colloidal quantum dots in polar solvents using a microemulsion-assisted method. Physical Chemistry Chemical Physics, 19(3):1999-2007, 2017.

[2] S. Alayoglu, P. Zavalij, B. Eichhorn, Q. Wang, A. I. Frenkel and P. Chupas. Structural and Architectural Evaluation of Bimetallic Nanoparticles: A Case Study of Pt-Ru Core-Shell and Alloy Nanoparticles. ACS Nano, 3(10):3127-3137, 2009.

[3] D.K. Avasthi and G.K. Mehta. Swift Heavy Ions for Materials Engineering and Nanostructuring, Springer Series in Materials Science Vol. 145. Springer-Verlag, Berlin, Germany, 2011.

[4] P. Bleuet, P. Cloetens, P. Gergaud, D. Mariolle, N. Chevalier, R. Tucoulou, J. Susini and A. Chabli. A hard x-ray nanoprobe for scanning and projection nanotomography. Rev. Sci. Instrum., 80, 056101:1-3, 2009

[5] T. Buonassisi, A.A. Istratov, M.A. Marcus, B. Lai, Z.H. Cai, S.M. Heald and E.R. Weber. Engineering metal-impurity nanodefects for low-cost solar cells. Nat. Mater., 4(9):676-679, 2005.

[6] M.Y. Chiu, U.S. Jeng, C.H. Su, K.S. Liang and K.H. Wei. Simultaneous Use of Small- and Wide-Angle X-ray Techniques to Analyze Nanometerscale Phase Separation in Polymer Heterojunction Solar Cells. Adv. Mater. 20(13):25732578, 2008.

[7] M. Cotte, J. Szlachetko, S. Lahlil, M. Salome, V. A. Sole, I. Biron, and J. Susini. Coupling a wavelength dispersive spectrometer with a synchrotron-based X-ray microscope: A winning combination for micro-X-ray fluorescence and micro-XANES analyses of complex artistic materials. J. Anal. At. Spectrom. 26:1051-1059, 2011.

[8] M. Cotte, A. Genty-Vincent, K. Janssens, J. Susini. Applications of synchrotron X-ray nano-probes in the field of cultural heritage. Comptes Rendus Physique, 19(7):575-588, 2018.

[9] C.-H. Cui and S.-H. Yu. Engineering Interface and Surface of Noble Metal Nanoparticle Nanotubes toward Enhanced Catalytic Activity for Fuel Cell Applications. Accounts of Chemical Research, 46(7):1427-1437, 2013.

[10] V. De Andrade, J. Thieme, P. Northrup, Y. Yao, A. Lanzirotti, P. Eng and Q. Shen. The sub-micron resolution x-ray spectroscopy beamline at NSLS-II. Nucl. Instrum. Meth. Phys. Res. Sect. A: Accelerat. Spectrom. Detect. Assoc. Equip. 649: 46-48, 2011.

[11] C.-L. Dong and L. Vayssieres. In Situ/Operando X-ray Spectroscopies for Advanced Investigation of Energy Materials. Chem. Eur. J. 24, 18356-18373, 2018.

[12] M.R. Fitzsimmons, S.D. Bader, J.A. Borchers, G.P. Felcher, J.K. Furdyna, A. Hoffmann, J.B. Kortright, I.K. Schuller, T.C. Schulthess, S.K. Sinha, M.F. Toney, D. Weller and S. Wolf. Neutron scattering studies of nanomagnetism and artificially structured materials. J. Magn. Magn. Mater., 271;103:146, 2004.

[13] A.I. Frenkel, A. Yevick, C. Cooper and R. Vasic. Modeling the Structure and Composition of Nanoparticles by Extended X-Ray Absorption Fine-Structure Spectroscopy. Annual Review of Analytical Chemistry, 4(1):23-39, 2011.

[14] A.I. Frenkel. Applications of extended X-ray absorption fine-structure spectroscopy to studies of bimetallic nanoparticle catalysts. Chem. Soc. Rev., 41:8163-8178, 2012. 
[15] F.J. García de Abajo. Optical excitations in electron microscopy. Rev. Mod. Phys., 82:209, 2010.

[16] M.B. Gawande, A. Goswami, T. Asefa, H. Guo, A.V. Biradar, D.-L. Peng, R. Zboril and R.S. Varma. Core-shell nanoparticles: synthesis and applications in catalysis and electrocatalysis. Chem. Soc. Rev., 44:7540-7590, 2015.

[17] P. Glatzel and U. Bergmann. High resolution 1s core hole X-ray spectroscopy in 3d transition metal complexes-electronic and structural information. Coord. Chem. Rev., 249:65-95, 2005.

${ }_{[18]}$ S. Guo, D. Li, H. Zhu, S. Zhang, N.M. Markovic, V.R. Stamenkovic, S. Sun. FePt and CoPt Nanowires as Efficient Catalysts for the Oxygen Reduction Reaction. Angewandte Chemie International Edition, 52(12):3465-3468, 2013.

[19] F. Hofmann, E. Tarleton, R.J. Harder, N.W. Phillips, P.-W. Ma, J.N. Clark, I.K. Robinson, B. Abbey, W. Liu, C.E. Beck. 3D lattice distortions and defect structures in ion-implanted nano-crystals. Scient. Rep. 7,45993,1-10, 2017.

[20] M. Holt, R. Harder, R. Winarski and V. Rose. Nanoscale hard X-ray microscopy methods for materials studies. Annu. Rev. Mater. Res., 43(1):183-211, 2013.

[21] C. Hoskins, Y. Min, M. Gueorguieva, C. McDougall, A. Volovick, P. Prentice, Z. Wang, A. Melzer, A. Cuschieri, L. Wang. Hybrid gold-iron oxide nanoparticles as a multifunctional platform for biomedical application. Journal of Nanobiotechnology, 10(1):27-39, 2012.

[22] G.E. Ice, J.D. Budai, J.W.L. Pang. The race to X-ray microbeam and nanobeam science. Science, 334(6060):12341239, 2011.

[23] L.-Y. Jiang, X.-X. Lin, A.-J. Wang, J. Yuan, J.-J Feng and X.-S. Li. Facile solvothermal synthesis of monodisperse Pt2.6Co1 nanoflowers with enhanced electrocatalytic activity towards oxygen reduction and hydrogen evolution reactions. Electrochimica Acta, 225(Supplement C):525-532, 2017

[24] R. Koester, J.-S. Hwang, D. Salomon, X. Chen, C. Bougerol, J.-P. Barnes, D.L. Dang, L. Rigutti, A.D. Bugallo, G. Jacopin, M. Tchernycheva, C. Durand and J. Eymery. M-Plane Core-Shell InGaN/GaN Multiple-Quantum-Wells on GaN Wires for Electroluminescent Devices. Nano Lett., (11):4839-4845, 2011.

[25] A. Kolmakov, S. Potluri, A. Barinov, T.O. Menteş, L. Gregoratti, M.A. Niño, A. Locatelli, M. Kiskinova. Spectromicroscopy for Addressing the Surface and Electron Transport Properties of Individual 1-D Nanostructures and Their Networks. ACS Nano 2(10):1993-2000, 2008.

[26] G. Krylova, N.M. Dimitrijevic, D.V. Talapin, J.R. Guest, H. Borchert, A. Lobo, T. Rajh and E.V. Shevchenko. Probing the Surface of Transition-Metal Nanocrystals by Chemiluminesence. Journal of the American Chemical Society, 132(26):9102-9110, 2010.

[27] G. Krylova, L.J. Giovanetti, F.G. Requejo, N.M. Dimitrijevic, A. Prakapenka and E.V. Shevchenko. Study of Nucleation and Growth Mechanism of the Metallic Nanodumbbells. Journal of the American Chemical Society, 134(9):43844392, 2012.

[28] W.M. Kwiatek and J.P. Glatzel. XAFS17 Highlights XAS and Related Techniques. Synchrotron Radiation News, 32(1):15-17, 2019

[29] J. Leveneur, G.I.N. Waterhouse, J. Kennedy, J.B. Metson and D.R.G. Mitchell. Nucleation and Growth of Fe Nanoparticles in SiO2: A TEM, XPS, and Fe L-Edge XANES Investigation. Phys. Chem. C, 115(43):20978-20985, 2011.

[30] Q. Li, L. Wu, G. Wu, D. Su, H. Lv, S. Zhang, W. Zhu, A. Casimir, H. Zhu, A. Mendoza-Garcia and S. Sun. New Approach to Fully Ordered fct-FePt Nanoparticles for Much Enhanced Electrocatalysis in Acid. Nano Letters, 15(4):2468-2473, 2015

[31] M.D. Mizrahi, G. Krylova, L.J. Giovanetti, J.M. Ramallo-López, Y. Liu, E.V. Shevchenko and F.G. Requejo. Unexpected compositional and structural modification of CoPt3 nanoparticles by extensive surface purification. Nanoscale, 10:6382-6392, 2018.

[32] G. Martinez-Criado, J. Segura-Ruiz, M. H.Chu, R.Tucoulou, I.Lopez, E. Nogales, B. Mendez and J. Piqueras. Crossed Ga2O3/SnO2 Multiwire Architecture: A Local Structure Study with Nanometer Resolution. Nano Lett. 14(10):5479:5487, 2014. 
[33] G. Martínez-Criado, J. Villanova, R. Tucoulou, D. Salomon, J.-P. Suuronen, S. Labouré, C. Guilloud, V. Valls, R. Barrett, E. Gagliardini, Y. Dabin, R. Baker, S. Bohic, C. Cohen and J. Morse. ID16B: a hard X-ray nanoprobe beamline at the ESRF for nano-analysis. J. Synchrotron Rad., 23:344-352, 2016.

[34] L. Mino, E. Borfecchia, J. Segura-Ruiz, C. Giannini, G. Martinez-Criado and C. Lamberti. Materials characterization by synchrotron x-ray microprobes and nanoprobes. Rev. Mod. Phys., 90(2):025007, 2018.

[35] D.E. Morrison, J.B. Aitken, M.D. de Jonge, J.A. Ioppolo, H.H. Harris, L.M. Rendina. High mitochondrial accumulation of new gadolinium(III) agents within tumour cells. Chem. Commun. 50:2252-2254, 2014.

[36] S. Mourdikoudis, R.M. Pallares and N.T.K. Thanh. Characterization techniques for nanoparticles: Comparison and complementarity upon studying nanoparticle properties. Nanoscale, 10:12871-12934, 2018.

[37] National Nanotechnology Initiative. X-rays and Neutrons: Essential Tools for Nanoscience Research. Report of the National Nanotechnology Initiative Workshop, Washington, 2005.

[38] E. Nazaretski, K. Lauer, H. Yan, N. Bouet, J. Zhou, R. Conley and X. Huang. Pushing the limits: an instrument for hard X-ray imaging below $20 \mathrm{~nm}$. J. Synchrotron Radiat., 22:336-341, 2015.

[39] E. Nazaretski, H. Yan, K. Lauer, N. Bouet, X. Huang, W. Xu, J. Zhou, D. Shu, Y. Hwu and Y. S. Chu. Design and performance of an X-ray scanning microscope at the Hard X-ray Nanoprobe beamline of NSLS-II. J. Synchrotron Rad., 24:1113-1119, 2017.

[40] S.A. Nepijko, E. Pippel and J. Woltersdorf. Dependence of lattice parameter on particle size. Physica status solidi (a), 61(2):469-475, 1980.

[41] D.A. Neumann. Neutron scattering and hydrogenous materials. Mater. Today 9:34-41, 2006.

[42] J.L. Provis, V. Rose, S.A. Bernal and J.S.J. van Deventer. High-Resolution Nanoprobe X-ray Fluorescence Characterization of Heterogeneous Calcium and Heavy Metal Distributions in Alkali-Activated Fly Ash. Langmuir, 25(19):11897-11904, 2009.

[43] J.M. Ramallo-Lopez, L. Giovanetti, A.F. Craievich, F.C. Vicentin, M. Marin-Almazo, M. Jose-Yacaman and F.G. Requejo. XAFS, SAXS and HREM characterization of Pd nanoparticles capped with n-alkyl thiol molecules. Physica B, 389:150-154, 2007.

[44] M. Salmeron. From Surfaces to Interfaces: Ambient Pressure XPS and Beyond. Top. Catal., 61(20):2044-2051, 2018.

[45] C. Sanchez-Cano, D. Gianolio, I. Romero-Canelon, R. Tucoulou and P.J. Sadler. Nanofocused synchrotron X-ray absorption studies of the intracellular redox state of an organometallic complex in cancer cells. Chem. Commun., 55:7065-7068, 2019.

[46] S. Schorr. The crystal structure of kesterite type compounds: A neutron and X-ray diffraction study. Solar Energy Mater. Solar Cells, 95(6):1482-1488, 2011.

[47] E. Secco, H.T. Mengistu, J. Segura-Ruíz, G. Martínez-Criado, A. García-Cristóbal, A. Cantarero, B. Foltynski, H. Behmenburg, C. Giesen, M. Heuken, N. Garro. Elemental Distribution and Structural Characterization of GaN/InGaN Core-Shell Single Nanowires by Hard X-ray Synchrotron Nanoprobes. Nanomaterials, 9(5):691-1,11, 2019.

[48] D. A. Shapiro, Y.-S. Yu, T. Tyliszczak, J. Cabana, R. Celestre, W. Chao and K. Kaznatcheev. Chemical composition mapping with nanometre resolution by soft x-ray microscopy. Nat. Photon., 8:765-769, 2014.

[49] J. Singh, C. Lamberti and J.A. van Bokhoven. Advanced X-ray absorption and emission spectroscopy: in situ catalytic studies. Chem. Soc. Rev., 39:4754-4766, 2010.

[50] V.R. Stamenkovic, B.S. Mun, K.J.J. Mayrhofer, P.N. Ross and N.M. Markovic. Effect of Surface Composition on Electronic Structure, Stability, and Electrocatalytic Properties of Pt-Transition Metal Alloys: Pt-Skin versus Pt-Skeleton Surfaces. Journal of the American Chemical Society 128(27):8813-8819, 2006.

[51] M. Suzuki. Hard-X-ray magnetic microscopy and local magnetization analysis using synchrotron radiation. Microscopy, 63(1):14-15, 2014. 
[52] F. Tao, M. E. Grass, Y. Zhang, D. R. Butcher, J. R. Renzas, Z. Liu, J. Y. Chung, B. S. Mun, M. Salmeron and G. A. Somorjai. Reaction-Driven Restructuring of Rh-Pd and Pt-Pd Core-Shell Nanoparticles. Science, 322:932-934, 2008.

[53] H.C.N. Tolentino, M.M. Soares, C.A. Perez, F.C. Vicentin, D.B. Abdala, D. Galante, V. de C. Teixeira, D.H.C. de Araújo and H. Westfahl Jr.. CARNAÚBA: The Coherent X-Ray Nanoprobe Beamline for the Brazilian Synchrotron SIRIUS/LNLS. J. Phys.: Conf. Ser., 849:012057, 2017.

[54] H.C.N. Tolentino, M.M. Soares, F.M.C. Silva, J.H. Rezende, D. Puglia, A. Bordin, M.S. Silva and R.R. Geraldes. Innovative instruments based on cryogenically cooled silicon crystals for the CARNAÚBA beamline at Sirius-LNLS. AIP Conference Proceedings, 2054:060026, 2019.

[55] H.C.N. Tolentino, R.R. Geraldes, et al. TARUMÃ station for the CARNAUBA beamline at SIRIUS/LNLS. Proc. SPIE 11112, X-Ray Nanoimaging: Instruments and Methods IV, 1111206, 2019.

[56] A. Ulvestad, A. Singer, J.N. Clark, H.M. Cho, J.-W. Kim, R. Harder, J. Maser, Y.S. Meng and O.G. Shpyrko. BATTERIES. Topological defect dynamics in operando battery nanoparticles. Science, 348(6241):1344-1347, 2015.

[57] D.F. van der Vliet, C. Wang, D. Li, A.P. Paulikas, J. Greeley, R.B. Rankin, D. Strmcnik, D. Tripkovic, N.M. Markovic, V.R. Stamenkovic. Unique Electrochemical Adsorption Properties of Pt-Skin Surfaces. Angewandte Chemie, 124(13):3193-3196, 2012.

[58] M.L. Vera, A. Cánneva, C. Huck-Iriart, F.G. Requejo, M.C. Gonzalez, M.L. Dell'Arciprete, A. Calvo. Fluorescent silica nanoparticles with chemically reactive surface: Controlling spatial distribution in one-step synthesis. Journal of colloid and interface science 496:456-464, 2017.

[59] B. Vianne, M.I. Richard, S. Escoubas, S. Labat, T. Schuelli, G. Chahine, V. Fiori and O. Thomas. Through-silicon via-induced strain distribution in silicon interposer. Appl. Phys. Lett., 106(14):141905,1-5, 2015.

[60] J. Villanova, J. Segura-Ruiz, T. Lafford and G. Martínez-Criado. Synchrotron microanalysis techniques applied to potential photovoltaic materials. Journal of synchrotron radiation, 19: 521-524, 2012.

[61] Z. L. Wang, T. S. Ahmad and M. A. El-Sayed. Steps, ledges and kinks on the surfaces of platinum nanoparticles of different shapes. Surf. Sci., 380:302-310, 1997

[62] R.P. Winarski, M.V. Holt, V. Rose, P. Fuesz, D. Carbaugh, C. Benson and D. Shu. A hard x-ray nanoprobe beamline for nanoscale microscopy. J. Synchrotron Radiat., 19:1056-1060, 2012.

[63] L.E. Wu, A. Levina, H.H. Harris, Z. Cai, B. Lai, S. Vogt, D.E. James, P.A. Lay. Carcinogenic Chromium(VI) Compounds Formed by Intracellular Oxidation of Chromium(III) Dietary Supplements by Adipocytes. Angew. Chem., 128:1774-1777, 2016.

[64] L. Wu, A. Mendoza-Garcia, Q. Li and S. Sun. Organic Phase Syntheses of Magnetic Nanoparticles and Their Applications. Chemical Reviews 55(5):1742-1745, 2016.

[65] H.L. Xin, S. Alayoglu, R. Tao, A. Genc, C.-M. Wang, L. Kovarik, E.A. Stach, L.-W. Wang, M. Salmeron, G.A. Somorjai and H. Zheng. Revealing the Atomic Restructuring of Pt-Co Nanoparticles. Nano Letters, 14(6):3203-3207, 2014.

[66] L. Yan, S. Jahangir, S.A. Wight, B. Nikoobakht, P. Bhattacharya and J.M. Millunchick. Structural and Optical Properties of Disc-in-Wire InGaN/GaN LEDs. Nano Lett., 15(3):1535-1539, 2015.

[67] Y. Yao and J.A. van Kan. Automatic beam focusing in the 2nd generation PBW line at sub-10 nm line resolution. Nucl. Instrum. Methods Phys. Res., Sect. B, 348:203-208, 2015.

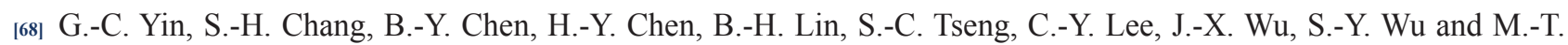
Tang. X-ray nanoprobe project at Taiwan Photon Source. AIP Conference Proceedings, 1741:030004, 2016.

[69] K. Zhang, Z. Zhao, Z. Wu and Y. Zhou. Synthesis and detection the oxidization of Co cores of Co@SiO2 core-shell nanoparticles by in situ XRD and EXAFS. Nanoscale Res. Lett., 10:37-46, 2015.

[70] J.F. Ziegler and J.P. Biersack. Treatise on Heavy-Ion Science, Astrophysics, Chemistry, and Condensed Matter, Vol. 6. D. A. Bromley. Springer US, Boston, MA, USA, 1985. 


\section{Bio}

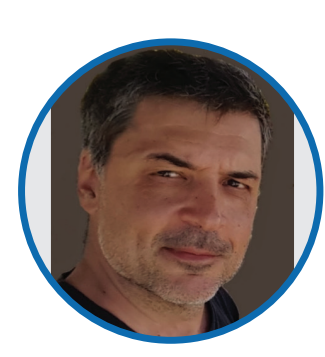

\section{Félix G. Requejo}

Professor at the National University of La Plata (UNLP, Argentina) and Researcher at CONICET, he obtained his $\mathrm{PhD}$ in Physics from UNLP in 1993. He was a postdoctoral fellow at the Lawrence Berkeley National Laboratory (CA, USA) in 2000 under the supervision of Prof. M.B. Salmeron. Back in Argentina in 2003, he started to work in characterization of nanomaterials, employing techniques derived from the use of synchrotron radiation. Prof. Requejo has published over 130 articles in international journals. He led more than hundred research proposals at different synchrotron laboratories around the world and formed several Ph.D. students. He received the "Dra. María Cristina Giordano" Prize in 2010 and the KONEX Prize in Nanotechnology in 2013. 